\title{
THE STRUCTURE OF AN EVEN LIAISON CLASS
}

\author{
GIORGIO BOLONDI AND JUAN C. MIGLIORE
}

\begin{abstract}
We describe a structure called the Lazarsfeld-Rao property for even liaison classes in projective space. This property holds for many even liaison classes of curves in $\mathbf{P}^{3}$. We give a procedure for showing that an even liaison class in codimension 2 possesses this property, and we prove it for a family of even liaison classes in codimension 2 in any $\mathbf{P}^{n}, n \geq 3$. However, we conjecture that it in fact holds for every even liaison class in codimension 2, so we want to give consequences for an even liaison class that possesses this property.

The main element in describing this structure is the notion of a basic double link. The Lazarsfeld-Rao property says that there exist minimal elements of the even liaison class and that any element of the even liaison class can be deformed to a curve obtained by a sequence of basic double links beginning with any minimal element. We show that there is a unique standard type of sequence for any given element of the even liaison class. As a result, we can express the even liaison class as a disjoint union of irreducible nonempty families parameterized by certain finite sequences of integers. The standard numerical invariants of the elements of any family can be computed from the associated sequence of integers. We apply this to surfaces in $\mathbf{P}^{4}$.

Our main tool for these results is a deformation technique related to liaison in codimension 2. We also study Schwartau's procedure of Liaison Addition in codimension 2 from the point of view of vector bundles. Using this, we give a different sort of structure for an even liaison class with the Lazarsfeld-Rao property.
\end{abstract}

\section{INTRODUCTION}

The question of parameterizing the even liaison classes in codimension 2 in projective space $\mathbf{P}^{n}$ was solved by Rao $[R]$, while the problem in higher codimension remains open. Equally interesting, however, is the question of describing the structure of an even liaison class. This was first considered by Lazarsfeld and Rao in [LR], where they showed that for a curve $C$ in $\mathbf{P}^{3}$ not lying on a surface of degree $e(C)+3$, the entire even liaison class of $C$ can be built up from $C$ by a process called basic double lilnkage, and then deforming. Although this hypothesis of [LR] is very strong and one expects that most even liaison classes do not possess such a curve, it was conjectured in [BM2] that

Received by the editors April 18, 1988.

1980 Mathematics Subject Classification (1985 Revision). Primary 14J10; Secondary 14C10.

Work by G. Bolondi is partially supported by MPI.

This material is based on work done while J. C. Migliore was supported by the North Atlantic Treaty Organization under a grant awarded in 1987. 
precisely the same structure continues to hold for every even liaison class of curves in $\mathbf{P}^{3}$. This structure was given the name LR-property. A procedure for verifying the LR-property was given in [BM2], and as an application it was shown that at least every Buchsbaum even liaison class possesses this property.

The present paper is an attempt to extend these ideas to $\mathbf{P}^{n}$. In the first section we show that in any codimension (except 1 and $n$ ) all the basic ideas used for curves in $\mathbf{P}^{3}$ (basic double linkage, shift, etc.) continue to make sense, and so the LR-property can be defined in this generality (Definition 1.8). Not surprisingly, though, the problem seems much more tractable in codimension 2 and so the remaining sections consider this case.

If $\S 2$ we first recall the connection between even liaison in codimension 2 and vector bundles on $\mathbf{P}^{n}[\mathrm{R}]$. We then show how this point of view can be applied to Schwartau's procedure of Liaison Addition [Sw]. The main result here is Proposition 2.7. This will be used again in $\S 5$.

In $\S 3$ we extend to $\mathbf{P}^{n}$ the ideas introduced in [BM2]. That is, we show that with essentially the same hypotheses used for curves in $\mathbf{P}^{3}$ we can produce the deformations (e.g., Proposition 3.1) needed for the LR-property. Furthermore, the inductive approach introduced in [BM2] to verify the LR-property for curves in $\mathbf{P}^{3}$ continues to work in codimension 2 in $\mathbf{P}^{n}$ (Theorem 3.10). Conjecturally, the LR-property should hold for all even liaison classes (at least in codimension 2). Hence it will be of interest to describe consequences of the LR-property. A potential problem, though, is that in practice we will use a technical condition to derive these consequences, rather than the LR-property itself. Happily, we are able to prove that this condition is equivalent to the LR-property (Proposition 3.11).

$\S 4$ uses the inductive approach described in $\S 3$ to verify the LR-property for a family of even liaison classes of two-codimensional subschemes of $\mathbf{P}^{n}$ $(n \geq 3)$, thus providing the first examples of this property beyond curves in $\mathbf{P}^{3}$. Namely, we consider the two-codimensional subschemes $X$ of $\mathbf{P}^{n}$ with only one intermediate cohomology group $h^{i}\left(\mathbf{P}^{n}, \mathscr{I}_{X}(t)\right)=p$ different from zero, $1 \leq i \leq n-2$.

In $\S 5$ we first give an in-depth study of the notion of basic double linkage (in codimension 2). The main result in this regard (Corollary 5.3) is that without loss of generality we can always assume that a sequence of basic double linkages is performed in a surprisingly simple way. Furthermore, this simplification is uniquely determined. This allows us to describe an even liaison class with the LR-property as a union of irreducible disjoint families, parameterized by certain finite sequences of integers (Corollary 5.7).

For example, we consider again the subschemes of $\mathbf{P}^{n}$ studied in $\S 4$. These are distributed into disjoint (nonvoid) irreducible families $\mathscr{F}_{\left(i ; p ; b ; g_{2}, \ldots, g_{r}\right)}$, with $1 \leq i \leq n-2, p \geq 1, b \geq 0, r \geq 1, \alpha<g_{2}<g_{3}<\cdots<g_{r}$, where $\alpha$ is an integer depending on $i, n$, and $p$, and $r$ is an integer that we assume to be 1 if there are no $g_{i}$ 's. We show, if $n=4$, how to determine the degree, 
the arithmetic genus, and the sectional genus (and other numerical invariants) of a surface belonging to $\mathscr{F}_{\left(i ; p ; b ; g_{2}, \ldots, g_{r}\right)}$. For instance, the general projection to $\mathbf{P}^{4}$ of the Veronese surface in $\mathbf{P}^{5}$ is in $\mathscr{F}_{(1 ; 1 ; 0)}$, and $r=1$. Note that these surfaces are the simplest example (from the cohomological point of view) of nonarithmetically Cohen-Macaulay surfaces in $\mathbf{P}^{4}$.

Another application (Theorem 5.11) is to show that given any two-codimensional subscheme $X$ of $\mathbf{P}^{n}$, any sequence of a basic double linkages beginning with $X$ is equivalent to a single liaison addition of $X$ with a suitable arithmetically Cohen-Macaulay subscheme. This gives an alternative and possibly simpler way of "bulding up" an even liaison class possessing the LR-property.

Acknowledgment. The authors thank the Department of Mathematics of the University of Trento (Italy), for its hospitality during the preparation of this paper.

\section{Preliminaries}

Let $k$ be an algebraically closed field, $S=k\left[X_{0}, \ldots, X_{n}\right], \mathbf{P}^{n}=\mathbf{P}_{k}^{n}$. All subschemes of $\mathbf{P}^{n}$ which we will consider will be assumed to be locally CohenMacaulay and equidimensional. If two subschemes $V_{1}$ and $V_{2}$ are directly linked by a complete intersection $X$, we will write $V_{1} \sim_{X} V_{2}$. If $\mathscr{F}$ is a sheaf on $\mathbf{P}^{n}$ we denote by $H_{*}^{i}(\mathscr{F})$ the direct sum $\bigoplus_{j \in \mathbf{Z}} H^{i}\left(\mathbf{P}^{n}, \mathscr{F}(j)\right)$. Also, $h^{i}\left(\mathbf{P}^{n}, \mathscr{F}\right)=\operatorname{dim}_{k} H^{i}\left(\mathbf{P}^{n}, \mathscr{F}\right)$.

Let $V$ be an $r$-dimensional subscheme of $\mathbf{P}^{n}$, for $1 \leq r \leq n-2$. Let $\left(M^{i}\right)(V)=H_{*}^{i}\left(\mathscr{J}_{V}\right)$ for $1 \leq i \leq r$. For each $i,\left(M^{i}\right)(V)$ is a graded $S$-module of finite length. $V$ is arithmetically Cohen-Macaulay if and only if $\left(M^{i}\right)(V)=0$ for all $1 \leq i \leq r$. Therefore, unless specifically noted otherwise, we henceforth assume that all schemes in question are not arithmetically Cohen-Macaulay, i.e., that we always have at least one nonzero module to work with. If $r=1$ then $\left(M^{1}\right)(V)$ is the Hartshorne-Rao module of $V$. The collection of all $\left(M^{i}\right)(V)$ is an invariant of the even liaison class of $V$ (up to shifts), as a result of the following theorem and corollary:

Theorem 1.1 [S, M2]. Let $V_{1}, V_{2}$ be r-dimensional subschemes of $\mathbf{P}^{n}$. Assume that $V_{1} \sim_{X} V_{2}$ where $I_{X}=\left(F_{1}, \ldots, F_{n-r}\right)$, and $\operatorname{deg} F_{i}=d_{i}$. Let $d=d_{1}+\cdots+$ $d_{n-r}$. Then $\left(M^{r-i+1}\right)\left(V_{1}\right) \cong\left(M^{i}\right)\left(V_{2}\right)^{\vee}(n+1-d)$ for all $1 \leq i \leq r$.

Corollary 1.2. If $V_{1}, V_{2}$ are evenly linked, then for some fixed integer $p$ we have

$$
\left(M^{i}\right)\left(V_{1}\right)(p) \cong\left(M^{i}\right)\left(V_{2}\right)
$$

for each $1 \leq i \leq r$.

Using this, we would like to partition the even liaison class of any scheme $V$ according to the shift of the associated modules. The first observation is that, as in the case of curves in $\mathbf{P}^{3}$, there is a notion of a minimal (leftward) shift. 
Recall the following:

Proposition 1.3 [M1, Sw]. Let $M$ be a nonzero graded $k\left[X_{0}, X_{1}, X_{2}, X_{3}\right]$ module of finite length. A necessary condition for $M$ to be the HartshorneRao module of a curve is that some nonzero component of $M$ be in degree zero or more. (This condition is not sufficient.)

This implies that if we shift $M$ enough leftward, it cannot be the HartshorneRao module of a curve and so there must be a leftmost shift. We want to generalize this to arbitrary subschemes of $\mathbf{P}^{n}$.

Let $V \subset \mathbf{P}^{n}$ be a subscheme of dimension $r(1 \leq r \leq n-2)$. As in [M1], we now show that the modules $\left(M^{i}\right)(V)$ cannot shift indefinitely leftward.

Let $\mathscr{L}$ be the even liaison class of $V$ and let us write

$$
c(\mathscr{L})=\left\{i \mid 1 \leq i \leq r,\left(M^{i}\right)(X) \neq 0 \text { if } X \in \mathscr{L}\right\} .
$$

(By hypothesis, $c(\mathscr{L}) \neq \varnothing$.)

Proposition 1.4. Iet $\mathscr{L}$ be an even liaison class of dimension $r$ subschemes in $\mathbf{P}^{n}(1 \leq r \leq n-2)$. Then there exists $X \in \mathscr{L}$ such that, for all $V \in \mathscr{L}$ and for all $i(1 \leq i \leq r)$, we have

$$
M^{i}(V) \cong M^{i}(X)(-d) \text { for some } d \geq 0 \text {. }
$$

Proof. If $n=3$ then the only possibility is covered by Proposition 1.3. Let $W \in \mathscr{L}, m=\min \{c(\mathscr{L})\}$, and $v(W)=\max \left\{t \mid H^{m}\left(\mathbf{P}^{n}, \mathscr{J}_{W}(t)\right) \neq 0\right\}$. Choosing a general hyperplane $H \subset \mathbf{P}^{n}$ and considering the sequence

$$
0 \rightarrow \mathscr{I}_{W^{\prime}}(-1) \rightarrow \mathscr{I}_{W} \rightarrow \mathscr{I}_{W^{\prime} \cap H} \rightarrow 0
$$

we get $h^{m-1}\left(H, \mathscr{I}_{W \cap H}(v(W)+1)\right) \neq 0$. If $m=1$, this implies that $v(W) \geq 0$. If $m>1$, then $h^{m-1}\left(H, \mathscr{J}_{W \cap H}(t)\right)=0$ for $t>v(W)+1$, and $h^{i}\left(H, \mathscr{I}_{W \cap H}(t)\right)$ $=0$ for all $t$ and all $i$ satisfying $1 \leq i \leq m-2$. By repeating this argument $m$ times (recall $m \leq r$ ), we get that $v(W) \geq 1-m$.

Hence the set $\{t \in \mathbf{Z} \mid t=v(W), W \in \mathscr{L}\}$ is bounded below. A subscheme $X \in \mathscr{L}$ for which $v(X)$ is minimal satisfies the thesis.

As has been done for curves in $\mathbf{P}^{3}$ (e.g., see [BM2]), we use the following notation for this partition:

Definition 1.5. Let $\mathscr{L}$ be an even liaison class of dimension $r$ subschemes of $\mathbf{P}^{n}$. Then $\mathscr{L}^{0}$ is the set of subschemes whose associated modules attain the leftmost possible shift. $\mathscr{L}^{h}$ is the set of subschemes whose associated modules are shifted $h$ places to the right of the leftmost shift.

Note that if $X \in \mathscr{L}^{0}$ and $Y \in \mathscr{L}^{h}$, then we have $M^{i}(X)(-h) \cong M^{i}(Y)$ for every $i, 1 \leq i \leq r$. Hence, in particular, for all $t \in \mathbf{Z}$ and for all $i, 1 \leq i \leq r$, we have $h^{i}\left(\mathbf{P}^{n}, \mathscr{I}_{X}(t)\right)=h^{i}\left(\mathbf{P}^{n}, \mathscr{I}_{Y}(t+h)\right)$.

We are grateful to R. Miro-Roig for pointing out the following example to us. 
Example 1.6. Let $V \subset \mathbf{P}^{4}$ be a general projection to $\mathbf{P}^{4}$ of the Veronese surface in $\mathbf{P}^{5}$, and let $\mathscr{L}$ be its even liaison class. It follows from the locally free resolution of $\mathscr{I}_{V}$

$$
0 \rightarrow \mathscr{O}_{\mathbf{P}^{4}}(-3) \oplus \mathscr{O}_{\mathbf{P}^{4}}(-3) \oplus \mathscr{O}_{\mathbf{P}^{4}}(-3) \rightarrow \Omega_{\mathbf{P}^{4}} \rightarrow \mathscr{I}_{V} \rightarrow 0
$$

(see [O1]) that $h^{1}\left(\mathbf{P}^{4}, \mathscr{J}_{V}(1)\right)=1$, and $h^{1}\left(\mathbf{P}^{4}, \mathscr{J}_{V}(t)\right)=0$ if $t \neq 1$. Also, $h^{2}\left(\mathscr{I}_{V}(t)\right)=0$ for all $t$. We check that in $\mathscr{L}$ there are no surfaces with $h^{1}\left(\mathbf{P}^{4}, \mathscr{J}_{X}(v)\right)=1$, and $v \leq 0$. Suppose otherwise. Taking a general hyperplane section, we get a curve $C$ with $h^{0}\left(\mathbf{P}^{3}, \mathscr{I}_{C}(v+1)\right) \geq 1$, and hence $h^{0}\left(\mathbf{P}^{3}, \mathscr{I}_{C}(1)\right) \geq 1$. But a curve contained in a plane is a complete intersection, hence it has $h^{1}\left(\mathbf{P}^{3}, \mathscr{I}_{C}(t)\right)=0$ for every $t$. This contradicts the fact that $h^{1}\left(\mathbf{P}^{3}, \mathscr{I}_{C}(v)\right)=1$ (from a usual exact sequence). Hence $V \in \mathscr{L}^{0}$.

This is a special case of the work in $\S 4$.

By definition, $\mathscr{L}^{0} \neq \varnothing$. In fact, $\mathscr{L}^{h} \neq \varnothing$ for each $h \geq 0$. This is an easy consequence of the notion of basic double linkage introduced (for curves in $\mathbf{P}^{3}$ ) in $[L R]$.

Definition 1.7. Let $V$ be an $r$-dimensional subscheme of $\mathbf{P}^{n}$ and $\mathscr{L}$ the even liaison class of $V$. Let $F_{1}, \ldots, F_{n-r} \in I_{V}$ be a regular sequence. Let $X_{1}, X_{2}$ be complete intersections, where $I_{X_{1}}=\left(F_{1}, \ldots, F_{n-r}\right)$ and $I_{X_{2}}=$ $\left(F_{1}, \ldots, F_{n-r-1}, A F_{n-r}\right)$ for a general form $A$ of degree $d$. Let $V \sim_{X_{1}} V_{1}$ and $V_{1} \sim{ }_{X_{2}} V_{2}$. Then $V_{2}$ is a basic double link of $V$. Note that if $V \in \mathscr{L}^{h}$ then $V_{2} \in \mathscr{L}^{h+d}$. Set theoretically (thinking of $F_{i}$ and $A$ as hypersurfaces) we have $V_{2}=V \cup\left(F_{1} \cap \cdots \cap F_{n-r-1} \cap A\right)$, and as ideals $I_{V_{2}}=A \cdot I_{V}+\left(F_{1}, \ldots, F_{n-r-1}\right)$. In particular, it is independent of $F_{n-r}$.

We shall see in $\S 3$ that at least in codimension 2 we lose no generality if we always assume that $\operatorname{deg} A=1$.

One of our main concerns in this paper is the problem of describing the structure of an even liaison class. This was first discussed for very special even liaison classes of curves in $\mathbf{P}^{3}$ by Lazarsfeld and Rao [LR]. In [BM2] it was conjectured that every even liaison class of curves in $\mathbf{P}^{3}$ has essentially the same structure as that described in [LR], and an approach was outlined to attack this conjecture. This approach was used to prove it at least for every even Buchsbaum liaison class of curves in $\mathbf{P}^{3}$.

However, it seems reasonable to ask if essentially the same structure holds in the generality we have been discussing thus far: dimension $r$ in $\mathbf{P}^{n} \quad(1 \leq$ $r \leq n-2)$. Thus we introduce the problem in this generality even though we restrict our attention to codimension 2 for the remainder of the paper.

Definition 1.8. Let $\mathscr{L}$ be an even liaison class of dimension $r$ subschemes of $\mathbf{P}^{n}$. We say that $\mathscr{L}$ has the LR-property if the following conditions hold: 
(a) If $V_{1}, V_{2} \in \mathscr{L}^{0}$ then there is a deformation from one to the other through subschemes all in $\mathscr{L}^{0}$.

(b) Given $V_{0} \in \mathscr{L}^{0}$ and $V \in \mathscr{L}^{h}(h \geq 1)$, there exists a sequence of subschemes $V_{0}, V_{1}, \ldots, V_{t}$ such that for all $i, 1 \leq i \leq t, V_{i}$ is a basic double link of $V_{i-1}$ and $V$ is a deformation of $V_{t}$ through subschemes all in $\mathscr{L}^{h}$.

The Lazarsfeld-Rao problem is to identify which even liaison classes $\mathscr{L}$ have the LR-property. The power of the LR-property is clear: it gives a strong description of the elements of the even liaison class. In the leftmost shift there is essentially only one element. And in subsequent shifts one can essentially describe all the possibilities simply by describing the possible sequences of basic double links which wind up in that shift. This is carefully worked out in $\S 5$.

For example, knowing that the LR-property holds for all Buchsbaum (nonarithmetically Cohen-Macaulay) even liaison classes in $\mathbf{P}^{3}$ [BM2], we can make the following list of all arithmetically Buchsbaum (nonarithmetically CohenMacaulay) curves $C$ in $\mathbf{P}^{3}$ of degree $\leq 8$. Here we denote $d=\operatorname{deg} C$, $g=p_{a}(C), i(C)=\operatorname{dim} M(C)$, and $h$ is the shift as described above.

\begin{tabular}{r|r|r|ll}
$d$ & $g$ & $i(C)$ & $h$ \\
\hline 2 & -1 & 1 & 0 \\
3 & - & - & $-\quad$ (does not exist) \\
4 & 0 & 1 & 1 \\
5 & 1 & 1 & 1 \\
6 & 3 & 1 & 1 \\
& 3 & 1 & 2 \\
7 & 4 & 1 & 2 \\
& 6 & 1 & 1 \\
8 & 8 & 1 & 3 \\
& 6 & 1 & 2 \\
& 5 & 2 & 0
\end{tabular}

A different approach to studying the structure of a liaison class (especially in the arithmetically Cohen-Macaulay case) was given in [HU].

\section{SchWartau's PROCEDURE OF LIAISON ADDITION IN CODIMENSION 2}

For the remainder of this paper we restrict ourselves to codimension 2 in $\mathbf{P}^{n}$. The question of when two codimension 2 subschemes of $\mathbf{P}^{n}$ are evenly linked was answered by Rao in [R]. We first recall his result. We will sometimes write $\mathscr{O}(a)$ for $\mathscr{O}_{\mathbf{P}^{n}}(a)(a \in \mathbf{Z})$. 
Definition 2.1. Let $\mathscr{F}_{1}, \mathscr{F}_{2}$ be vector bundles on $\mathbf{P}^{n}, \mathscr{F}_{1}$ and $\mathscr{F}_{2}$ are stably equivalent if

$$
\mathscr{F}_{1} \oplus \bigoplus_{i=1}^{p} \mathscr{O}\left(a_{i}\right) \cong \mathscr{F}_{2}(c) \oplus \bigoplus_{j=1}^{q} \mathscr{O}\left(b_{j}\right)
$$

for some $c, a_{i}, b_{j} \in \mathbf{Z}$ and $p, q$ nonnegative integers. This is an equivalence relation among bundles on $\mathbf{P}^{n}$.

Theorem $2.2[\mathrm{R}]$. In $\mathbf{P}^{n}(n \geq 2)$ the even liaison classes in codimension 2 are in bijective correspondence with the stable equivalence classes of vector bundles $\mathscr{F}$ on $\mathbf{P}^{n}$ with $H_{*}^{1}(\mathscr{F})=0$.

In fact, given $V \subset \mathbf{P}^{n}$ of codimension 2 , the vector bundle $\mathscr{F}$ associated to $V$ is obtained as follows. Begin with a free $S$-resolution of $I_{V}$ and sheafify. This produces an exact sequence

$$
0 \rightarrow \mathscr{F} \rightarrow \bigoplus_{i=1}^{m} \mathscr{O}\left(-c_{i}\right) \rightarrow \mathscr{J}_{V} \rightarrow 0
$$

where $\mathscr{F}$ is locally free since $V$ is locally Cohen-Macaulay of codimension 2 .

We can now relate this to our notion of shifts. Suppose that $V_{1}$ and $V_{2}$ are in the same even liaison class. It follows that we have exact sequences

$$
\begin{aligned}
& 0 \rightarrow \mathscr{F}_{1} \rightarrow \bigoplus \mathscr{O}\left(-c_{i}\right) \rightarrow \mathscr{I}_{V_{1}} \rightarrow 0, \\
& 0 \rightarrow \mathscr{F}_{2} \rightarrow \bigoplus \mathscr{O}\left(-d_{j}\right) \rightarrow \mathscr{I}_{V_{2}} \rightarrow 0,
\end{aligned}
$$

where $\mathscr{F}_{1}$ and $\mathscr{F}_{2}$ are locally free, $H_{*}^{1}\left(\mathscr{F}_{1}\right)=H_{*}^{1}\left(\mathscr{F}_{2}\right)=0$, and $\mathscr{F}_{1} \oplus A \cong$ $\mathscr{F}_{2}(c) \oplus B$, where $A$ and $B$ are direct sums of line bundles.

Lemma 2.3. $V_{1}$ and $V_{2}$ are in the same shift if and only if $c=0$.

Proof. We have $\left(M^{i}\right)\left(V_{1}\right) \cong H_{*}^{i+1}\left(\mathscr{F}_{1}\right)$ for $1 \leq i \leq n-2$, and similarly for $V_{2}$, $\mathscr{F}_{2}$. If $V_{1}$ and $V_{2}$ are in the same shift then $H_{*}^{i+1}\left(\mathscr{F}_{1}\right) \cong H_{*}^{i+1}\left(\mathscr{F}_{2}\right), 0 \leq i \leq n-2$ (since we also know $H_{*}^{1}\left(\mathscr{F}_{1}\right)=H_{*}^{1}\left(\mathscr{F}_{2}\right)=0$ ). But $H_{*}^{i+1}\left(\mathscr{F}_{1}\right) \cong H_{*}^{i+1}\left(\mathscr{F}_{1}(c)\right.$ ). Since we have assumed that at least one of these modules is nonzero, it follows that $c=0$.

Conversely, if $c=0$ then $H_{*}^{i+1}\left(\mathscr{F}_{1}\right) \cong H_{*}^{i+1}\left(\mathscr{F}_{2}\right)$ for $0 \leq i \leq n-2$, so they are in the same shift.

We now turn to Schwartau's procedure of Liaison Addition [Sw]. This procedure has been extremely important in the study of liaison of curves in $\mathbf{P}^{3}$, but it does not seem to have been applied yet to higher projective spaces. In fact, in [Sw] Schwartau makes the initial definitions and observations on the generality of codimension 2 subschemes of $\mathbf{P}^{n}$ and provides a powerful technique for constructing new liaison classes starting from known ones. We recall the relevant results, but now our point of view is of vector bundles and Theorem 2.2. As noted below, much of this section is a quotation from [Sw], simply adapted to our context. 
Definition 2.4 [Sw, Definition 4.9]. Let $X, Y$ be closed two-codimensional subschemes in $\mathbf{P}^{n}$ with total ideals $I_{X}$ and $I_{Y}$. Choose any hypersurfaces $\Sigma_{a}$ containing $X$ and $\Sigma_{b}$ containing $Y$ (with degrees $a$ and $b$, respectively), such that $\left(\Sigma_{a}, \Sigma_{b}\right)$ is a complete intersection (general choices suffice). The liaison addition of $X$ and $Y$ via $\Sigma_{a}$ and $\Sigma_{b}$ is the closed subscheme of $\mathbf{P}^{n}$ defined by the homogeneous ideal $\Sigma_{b} I_{X}+\Sigma_{a} I_{Y}$.

Theorem 2.5 [Sw, Theorem 50]. Let $X, Y$ be two-codimensional subschemes of $\mathbf{P}^{n}$, and let $Z$ be the liaison addition of $X$ and $Y$ via hypersurfaces $\Sigma_{a}$ and $\Sigma_{b}$. Then

(1) $\Sigma_{b} I_{X}+\Sigma_{a} I_{Y}=I_{Z}$, the total ideal of $Z$.

(2) $Z$ is two-codimensional.

(3) $\forall i \geq 3, \operatorname{Ext}_{S}^{i}\left(S / I_{Z}, S\right) \cong \operatorname{Ext}_{S}^{i}\left(S / I_{X}, S\right)(b) \oplus \operatorname{Ext}_{S}^{i}\left(S / I_{Y}, S\right)(a)$.

(4) $Z$ is locally Cohen-Macaulay and equidimensional if and only if $X$ and $Y$ are locally Cohen-Macaulay and equidimensional.

(5) Set-theoretically, $Z=X \cup Y \cup\left(\Sigma_{a} \cap \Sigma_{b}\right)$.

An immediate consequence is

Corollary 2.6 [Sw, Remark 56]. If $X$ and $Y$ are curves in $\mathbf{P}^{3}$ and $Z$ is the liaison addition of $X$ and $Y$ via $\Sigma_{a}$ and $\Sigma_{b}$, then $M(Z) \cong M(X)(-b) \oplus$ $M(Y)(-a)$.

Hence in liaison addition the Hartshorne-Rao modules sum (suitably shifted). The content of Theorem 2.5(3) is that the same is true for the modules $\left(M^{i}\right)(V)$ for codimension 2 in general. This also follows from Proposition 2.7.

Let us turn now to $\mathbf{P}^{n}$. The invariant characterizing a codimension 2 liaison class is an equivalence class of vector bundles (Theorem 2.2). It is natural to ask what happens to these invariants when a liaison addition is performed, and the answer is also natural.

Proposition 2.7. Let $X$ and $Y$ be two-codimensional subschemes of $\mathbf{P}^{n}$ equidimensional and locally Cohen-Macaulay, with locally free resolutions

$$
\begin{gathered}
0 \rightarrow \mathscr{F}_{1} \rightarrow P \rightarrow \mathscr{I}_{Y} \rightarrow 0, \\
0 \rightarrow \mathscr{F}_{2} \rightarrow Q \rightarrow \mathscr{I}_{X} \rightarrow 0
\end{gathered}
$$

$\left(P\right.$ and $Q$ are sums of line bundles, and $h^{1}\left(\mathbf{P}^{n}, \mathscr{F}_{1}(t)\right)=0=h^{1}\left(\mathbf{P}^{n}, \mathscr{F}_{2}(t)\right)$ for all $t)$. Let $Y$ be contained in a hypersurface $\Sigma_{t}$ of degree $t$ and $X$ be contained in a hypersurface $\Sigma_{r}$ of degree $r$, meeting properly, and let $Z$ be the liaison addition of $X$ and $Y$ via $\Sigma_{r}$ and $\Sigma_{t}$. Then $Z$ has a locally free resolution

$$
0 \rightarrow \mathscr{F}_{1}(-r) \oplus \mathscr{F}_{2}(-t) \oplus \mathscr{O}_{\mathbf{p}^{n}}(-r-t) \rightarrow P(-r) \oplus Q(-t) \rightarrow \mathscr{I}_{Z} \rightarrow 0,
$$

where $A$ and $B$ are sums of line bundles.

Moreover, there is an exact sequence

$$
0 \rightarrow \mathscr{O}_{\mathbf{P}^{n}}(-r-t) \rightarrow \mathscr{I}_{Y}(-r) \oplus \mathscr{I}_{X}(-t) \rightarrow \mathscr{I}_{Y} \rightarrow 0 .
$$


Proof. Let us denote by $C$ the complete intersection $\Sigma_{r} \cap \Sigma_{t}$. Let $F$ and $G$ denote polynomials defining the surfaces $\Sigma_{r}$ and $\Sigma_{t}$, respectively. So we have $I_{C}=(F, G)$. Note that $C$ is contained in $Z$. We first claim that there is an exact sequence

$$
0 \rightarrow \mathscr{O}_{Y}(-r) \oplus \mathscr{O}_{X}(-t) \rightarrow \mathscr{O}_{Z} \rightarrow \mathscr{O}_{C} \rightarrow 0
$$

Indeed, we have an exact sequence of modules

$$
0 \rightarrow \frac{(F, G)}{G \cdot I_{X}+F \cdot I_{Y}} \rightarrow \frac{S}{G \cdot I_{X}+F \cdot I_{Y}} \rightarrow \frac{S}{(F, G)} \rightarrow 0 .
$$

Our claim will follow by sheafification, once we prove that

$$
\left(S / I_{Y}\right)(-r) \oplus\left(S / I_{X}\right)(-t) \cong \frac{(F, G)}{G \cdot I_{X}+F \cdot I_{Y}} .
$$

The isomorphism $\varphi$ is given by $\varphi(A, B)=A F+B G$. We leave well-definedness and surjectivity to the reader. For injectivity, suppose that for some $A, B \in S$ we have $\varphi(A, B)=0$. Then $A F+B G=a F+b G$ for some $a \in I_{Y}$ and $b \in I_{X}$. So $F(A-a)=G(b-B)$. But $F$ and $G$ have no common components, so $A-a \in(G)$ and $b-B \in(F)$. That is, $A=a+c G$ and $B=b+d F$ for some $c, d \in S$. Therefore $A \in I_{Y}$ and $B \in I_{X}$. This proves the claim.

Having the exact sequence $(*)$, we now consider the commutative diagram

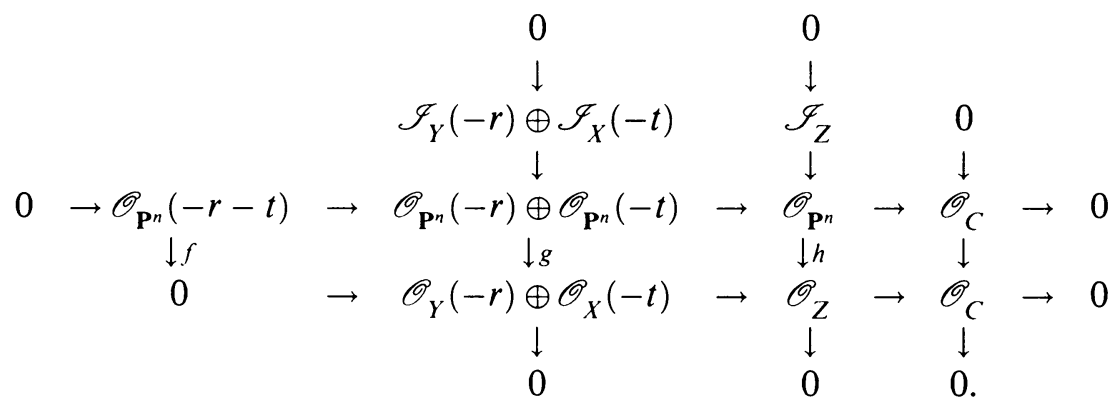

It is commutative since in both lines the horizontal arrows are the multiplications induced by $\Sigma_{r}$ and $\Sigma_{t}$. A diagram chase shows that there is an exact sequence of the kernels of $f, g$, and $h$ :

$$
0 \rightarrow \mathscr{O}_{\mathbf{P}^{n}}(-r-t) \rightarrow \mathscr{I}_{Y}(-r) \oplus \mathscr{I}_{X}(-t) \rightarrow \mathscr{I}_{Z} \rightarrow 0 .
$$

From the definition of $I_{Z}$ we see that there is a natural surjection

$$
P(-r) \oplus Q(-t) \rightarrow \mathscr{I}_{Z} \rightarrow 0 .
$$


(See the map $r$ in the commutative diagram below.) Let $\mathscr{K}$ be the kernel. We want to see what the relation is between $\mathscr{F}_{1}, \mathscr{F}_{2}$, and $\mathscr{K}$.

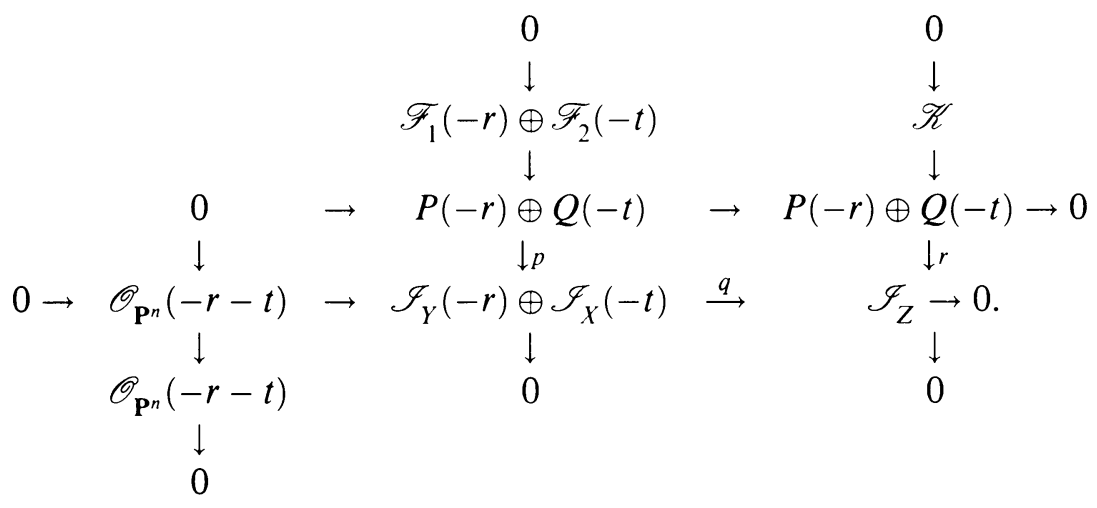

(The vertical map $r$ is just the composition of $p$ and $q$. Because of the way $Z$ is defined, $r$ gives the sheafification of a presentation of $I_{Z}$.) Then the snake lemma gives an exact sequence

$$
0 \rightarrow \mathscr{F}_{1}(-r) \oplus \mathscr{F}_{2}(-t) \rightarrow \mathscr{K} \rightarrow \mathscr{O}_{\mathbf{p}^{n}}(-r-t) \rightarrow 0,
$$

hence an element of $\operatorname{Ext}^{1}\left(\mathscr{O}_{\mathbf{p}^{n}}(-r-t), \mathscr{F}_{1}(-r) \oplus \mathscr{F}_{2}(-t)\right)$ [H, Exercise III.6.1]. But this group is zero, since

$$
\begin{aligned}
\operatorname{Ext}^{1}\left(\mathscr{O}_{\mathbf{p}^{n}}(-r-t), \mathscr{F}_{1}(-r) \oplus \mathscr{F}_{2}(-t)\right) & \cong \operatorname{Ext}^{1}\left(\mathscr{O}_{\mathbf{p}^{n}}, \mathscr{F}_{1}(t) \oplus \mathscr{F}_{2}(r)\right) \\
& \cong H^{1}\left(\mathbf{P}^{n}, \mathscr{F}_{1}(t) \oplus \mathscr{F}_{2}(r)\right) \\
& \cong H^{1}\left(\mathbf{P}^{n}, \mathscr{F}_{1}(t)\right) \oplus H^{1}\left(\mathbf{P}^{n}, \mathscr{F}_{2}(r)\right) \\
& =0 \text { by hypothesis. }
\end{aligned}
$$

Hence the sequence (\#) splits, and $\mathscr{K} \cong \mathscr{F}_{1}(-r) \oplus \mathscr{F}_{2}(-t) \oplus \mathscr{O}_{\mathbf{P}^{n}}(-r-t)$.

Corollary 2.8. The vector bundle corresponding to the liaison class of $Z$ (liaison addition of $X$ and $Y$ ) is in the same stable equivalence class of vector bundles as the direct sum of the vector bundles corresponding to $X$ and $Y$, suitably shifted.

Hence the operation on vector bundles corresponding to liaison addition is the direct sum (after shifting, and modulo stable equivalence).

A special case of liaison addition is the basic double linkage procedure that we saw in $\S 1$.

\section{Deformations AND the LR-PROBlem IN CODIMENSION 2}

In the previous section we saw that vector bundles $\mathscr{F}$ and, more precisely, exact sequences of the form

$$
0 \rightarrow \mathscr{F} \rightarrow \bigoplus \mathscr{O}\left(-c_{i}\right) \rightarrow \mathscr{I}_{V^{\prime}} \rightarrow 0
$$

are intimately related to liaison in codimension 2 . We now tie them in with the deformation results we shall need later. 
It is known that the curves in $\mathbf{P}^{3}$ with fixed cohomology structure form an irreducible family; that is to say, given two curves $X$ and $Y$ with the same cohomology in the sense that $h^{i}\left(\mathbf{P}^{3}, \mathscr{I}_{Y}(t)\right)=h^{i}\left(\mathbf{P}^{3}, \mathscr{I}_{X}(t)\right.$ for all $i, t$ and the multiplication on the $h^{1}$-modules is the same, then there exists an irreducible flat family of curves to which both $X$ and $Y$ belong [B]. This result depends on the fact that two such curves are in the same shift of the same even liaison class (if $X$ and $Y$ are arithmetically Cohen-Macaulay the result is well known [E]).

There is an analogous statement for codimension 2 subvarieties of $\mathbf{P}^{N}$. Again, we assume that our even liaison class is not arithmetically CohenMacaulay, since that result is already known, and in any case the notion of "shift" does not make sense.

Proposition 3.1. Let $\mathscr{L}$ be an even liaison class of two-codimensional subvarieties of $\mathbf{P}^{n}$, and let $X, Y \in \mathscr{L}^{h}$ such that

$$
h^{0}\left(\mathbf{P}^{n}, \mathscr{I}_{Y}(t)\right)=h^{0}\left(\mathbf{P}^{n}, \mathscr{I}_{X}(t)\right) \text { for all } t .
$$

Then there exists an irreducible flat family $\left\{X_{s}\right\}_{s \in S}$ of two-codimensional subvarieties of $\mathbf{P}^{n}$ to which both $X$ and $Y$ belong. Moreover, $S$ can be chosen so that for all $s \in S, X_{s} \in \mathscr{L}^{h}$ and $h^{0}\left(\mathbf{P}^{n}, \mathscr{I}_{X}(t)\right)=h^{0}\left(\mathbf{P}^{n}, \mathcal{J}_{X_{s}}(t)\right)$ for all $t$.

Proof. Let

$$
0 \rightarrow E \stackrel{f}{\rightarrow} \bigoplus_{1}^{r} \mathscr{O}_{\mathbf{P}^{n}}\left(-a_{i}\right) \rightarrow \mathscr{I}_{x} \rightarrow 0
$$

be a locally free resolution of $X$, with $H^{1}\left(\mathbf{P}^{n}, E(t)\right)=0$ for all $t$. Since $Y$ is evenly linked to $X$, a repeated use of [LR] gives a locally free resolution of $\mathscr{I}_{Y}$ :

$$
0 \rightarrow E(d) \oplus A \stackrel{g}{\longrightarrow} B \rightarrow \mathscr{I}_{Y} \rightarrow 0,
$$

where $A$ and $B$ are direct sums of line bundles. Since $Y$ is in the same shift as $X$, it follows that $d=0$ (using an argument similar to that in Lemma 2.3).

We get another locally free resolution of $\mathscr{I}_{X}$ from (1), by adding a trivial addendum:

$$
0 \rightarrow E \oplus A \stackrel{f \oplus I_{A}}{\longrightarrow} \bigoplus_{1}^{r} \mathscr{O}_{\mathbf{P}^{n}}\left(-a_{i}\right) \oplus A \rightarrow \mathscr{I}_{X} \rightarrow 0 .
$$

Let us rewrite (2) and (3) in the form

$$
\begin{aligned}
& 0 \rightarrow F \stackrel{u}{\rightarrow} \bigoplus_{1}^{m} \mathscr{O}_{\mathbf{P}^{n}}\left(-h_{i}\right) \rightarrow \mathscr{I}_{Y} \rightarrow 0, \\
& 0 \rightarrow F \stackrel{n}{\rightarrow} \bigoplus_{1}^{m} \mathscr{O}_{\mathbf{p}^{n}}\left(-k_{i}\right) \rightarrow \mathscr{I}_{X} \rightarrow 0,
\end{aligned}
$$


where $F=E \oplus A$ (and hence $H^{1}\left(\mathbf{P}^{n}, F(t)\right)=0$ for all $t$ ) and $m=r k F+1$.

From the long cohomology sequences associated to $\left(2^{\prime}\right)$ and $\left(3^{\prime}\right)$ we get

$$
\begin{aligned}
& 0 \rightarrow H^{0}\left(\mathbf{P}^{n}, F(t)\right) \rightarrow \bigoplus_{1}^{m} H^{0}\left(\mathbf{P}^{n}, \mathscr{O}_{\mathbf{P}^{n}}\left(t-h_{i}\right)\right) \rightarrow H^{0}\left(\mathbf{P}^{n}, \mathscr{J}_{Y}(t)\right) \rightarrow 0, \\
& 0 \rightarrow H^{0}\left(\mathbf{P}^{n}, F(t)\right) \rightarrow \bigoplus_{1}^{m} H^{0}\left(\mathbf{P}^{n}, \mathscr{O}_{\mathbf{P}^{n}}\left(t-k_{i}\right)\right) \rightarrow H^{0}\left(\mathbf{P}^{n}, \mathscr{I}_{X}(t)\right) \rightarrow 0 .
\end{aligned}
$$

Then from the hypothesis $h^{0}\left(\mathbf{P}^{n}, \mathscr{J}_{X}(t)\right)=h^{0}\left(\mathbf{P}^{n}, \mathscr{J}_{Y}(t)\right)$ for all $t$ we conclude that $h_{i}=k_{i}$ for every $i=1,2, \ldots, m$.

Hence, $u, v \in \operatorname{Hom}\left(F, \bigoplus_{1}^{m} \mathscr{O}_{\mathbf{p}^{n}}\left(-h_{i}\right)\right)$. Given $s \in k$ (the base field), let

$$
w_{s}=s u+(1-s) v \in \operatorname{Hom}\left(F, \bigoplus_{1}^{m} \mathscr{O}_{\mathbf{P}^{n}}\left(-h_{i}\right)\right) .
$$

Then if $s$ is general, the map of locally free sheaves $w_{s}$ has, as cokernel, the ideal sheaf $\mathcal{F}_{X_{s}}$ of a two-codimensional subvariety, and these subvarieties fit together in an irreducible flat family in $\mathbf{P}^{n}$ (see [K] and [LR]). Note that $X_{s}$ is in the same shift of the same liaison class as $X$ and $Y$ (thanks to Theorem 2.2 and Lemma 2.3), and it is trivial to check that

$$
h^{0}\left(\mathbf{P}^{n}, \mathscr{I}_{X}(t)\right)=h^{0}\left(\mathbf{P}^{n}, \mathscr{I}_{X_{s}}(t)\right) \text { for all } t .
$$

Let us say that two codimension 2 subvarieties of $\mathbf{P}^{n} X$ and $Y$ have the same postulation if $h^{0}\left(\mathbf{P}^{n}, \mathscr{I}_{X}(t)\right)=h^{0}\left(\mathbf{P}^{n}, \mathscr{J}_{Y}(t)\right)$ for all $t$. We can rephrase our proposition in the following form:

Corollary 3.2. The two-codimensional subvarieties of $\mathbf{P}^{n}$ belonging to the same shift of the same even liaison class, with fixed postulation, arm in an irreducible family.

Remark 3.3. The same proof works if we assume that $h^{n-1}\left(\mathbf{P}^{n}, \mathscr{I}_{X}(t)\right)=$ $h^{n-1}\left(\mathbf{P}^{n}, \mathscr{I}_{Y}(t)\right)$ for all $t$.

Another corollary of Proposition 3.1 is

Corollary 3.4. Let $X$ and $Y$ be two-codimensional subvarieties of $\mathbf{P}^{n}$ in the same shift of the same even liaison class, and let $H$ be a general hyperplane. If $X \cap H$ has the same Hilbert function as $Y \cap H$, then there is a deformation from $X$ to $Y$.

Proof. If $X \cap H$ and $Y \cap H$ have the same Hilbert function, and $X$ and $Y$ are in the same shift of the same liaison class, then $X$ and $Y$ have the same postulation. (The proof is identical to that given in [BM2] for the case of curves.) Then the result follows.

In fact, we can reduce the problem to points in $\mathbf{P}^{2}$. To do this, we first need a result which is of interest in itself. While intuitively natural, we are not aware of a proof in the literature. 
Lemma 3.5. Let $V_{1}$ and $V_{2}$ be two-codimensional subschemes of $\mathbf{P}^{n}$ which are directly linked by a complete intersection $X$. Let $H$ be a hyperplane which contains no components of either $V_{1}$ or $V_{2}$. Then $V_{1} \cap H$ is directly linked in $H=\mathbf{P}^{n-1}$ to $V_{2} \cap H$ by the complete intersection $X \cap H$.

Proof. Let $L$ be the linear form defining the hyperplane $H$. It should be noted that by $(-\cap H)$ we mean the geometric hyperplane section, in the sense of [GM1] - that is, the scheme associated to the saturation of the ideal obtained by modding by $L$.

Without loss of generality take $L=X_{0}$, so we have $S=k\left[X_{0}, \ldots, X_{n}\right]$ and we let $R=S /(L)=k\left[X_{0}, \ldots, X_{n-1}\right]$. By hypotheses we have $\left(I_{X}: I_{V_{1}}\right)=I_{V_{2}}$ in $S$. Passing to $R$, notice first that $I_{X \cap H}=\left(I_{X}\right) /\left(L \cdot I_{X}\right)$ since $X$ is arithmetically Cohen-Macaulay. Also, since

$$
I_{V_{i} \cap H} \supseteq\left(I_{V_{i}}\right) /\left(L \cdot I_{V_{i}}\right)=\left(I_{V_{i}}, L\right) /(L) \supseteq I_{X \cap H}
$$

in $R$ (by generality of $L$ ) we see that as schemes $V_{i} \cap H \subseteq X \cap H$, and certainly as sets $\left(V_{1} \cap H\right) \cup\left(V_{2} \cap H\right)=X \cap H$. Hence we have only to prove that $\left(I_{X \cap H}: I_{V_{1} \cap H}\right)=I_{V_{2} \cap H}$. Furthermore, both sides of the equality to be proved represent the saturated, homogeneous ideals of locally Cohen-Macaulay twocodimensional subschemes of $H$ of the same degree (namely $\operatorname{deg} X-\operatorname{deg} V_{1}$ ). Therefore it suffices to prove that $\left(I_{X \cap H}: I_{V_{1} \cap H}\right) \supseteq I_{V_{2} \cap H}$.

For any ideal $I$ we denote by $\bar{I}$ its saturation. In particular, we have

$$
\overline{\left(I_{V_{i}}, L\right) /(L)}=I_{V_{i} \cap H} \quad(i=1,2) .
$$

Now we will use the basic fact that if $I$ and $J$ are ideals with $I=\bar{I}$, then $(I: J)=(I: \bar{J})$. (This follows more or less immediately from the definitions.) As a result, $\left(I_{X \cap H}: I_{V_{1} \cap H}\right)=\left(I_{X \cap H}:\left[\left(I_{V_{1}}, L\right) /(L)\right]\right)$.

Now, we claim that

$$
\left(I_{X \cap H}:\left[\left(I_{V_{1}}, L\right) /(L)\right]\right)=\left(\left(I_{X}, L\right) /(L):\left[\left(I_{V_{1}}, L\right) /(L)\right]\right) \supseteq\left(I_{V_{2}}, L\right) /(L)
$$

(as ideals in $R=S /(L))$. In fact, let $f \in\left(I_{V_{2}}, L\right) /(L)$. Say $f=[F]$ where $F \in I_{V_{2}}$. By hypothesis, $F \cdot I_{V_{1}} \subseteq I_{X}$. Let $g \in\left(I_{V_{1}}, L\right) /(L), g=[G]$, where $G \in I_{V_{1}}$. In particular, $F G \in I_{X}$. Then $f g=[F G] \in\left(I_{X}, L\right) /(L)=I_{X \cap H}$. Therefore $f \in\left(I_{X \cap H}:\left[\left(I_{V_{1}}, L\right) /(L)\right]\right)$.

Combining, we get

$$
\left(I_{X \cap H}: I_{V_{1} \cap H}\right)=\left(I_{X \cap H}:\left[\left(I_{V_{1}}, L\right) /(L)\right]\right) \supseteq\left(I_{V_{2}}, L\right) /(L) .
$$

But $\left(I_{X \cap H}: I_{V_{1} \cap H}\right)$ is saturated, so it contains the saturation of $\left(I_{V_{2}}, L\right) /(L)$. That is, $\left(I_{X \cap H}: I_{V_{1} \cap H}\right) \supseteq I_{V_{2} \cap H}$, which was what we wanted to prove.

Corollary 3.6. Let $\Lambda$ be a general 2-plane in $\mathbf{P}^{n}$. Assume $V_{1}$ and $V_{2}$ are evenly linked and in the same shift. If $V_{1} \cap \Lambda$ and $V_{2} \cap \Lambda$ have the same Hilbert function, then there exists a deformation from one to the other as described in Proposition 3.1.

Proof. In general it is not the case that if we know the module structure of $\left(M^{i}\right)(V)=H_{*}^{i}\left(\mathbf{P}^{n}, \mathscr{J}_{V}\right)$ for all $i$ then we can deduce the module structure of 
$\left(M^{i}\right)(V \cap H)$. For example, if $\left(M^{i}\right)(V)$ is annihilated by the maximal ideal m of $S$ for all $i(1 \leq i \leq n-2)$, then the same may or may not be true for $\left(M^{i}\right)(V \cap H)$ (see [SV]). However, we have just seen that if $V_{1}$ and $V_{2}$ are evenly linked then so are $V_{1} \cap H$ and $V_{2} \cap H$. Hence $\left(M^{i}\right)\left(V_{1} \cap H\right) \cong\left(M^{i}\right)\left(V_{2} \cap H\right)$ for $1 \leq i \leq n-3$ since we have assumed the same shift. Similarly for successive hyperplane sections.

Now, say $\Lambda=H_{1} \cap \cdots \cap H_{n-2}$ where each $H_{i}$ is defined by the vanishing of a linear form $L_{i}$. Let $C_{i}=V_{i} \cap\left(H_{2} \cap \cdots \cap H_{n-2}\right)(i=1,2)$. Then $M\left(C_{1}\right) \cong M\left(C_{2}\right)$, so multiplication by the linear form $L_{1}$ produces the same homomorphism on each. Let $K_{j}$ be the kernel of the map

$$
M\left(C_{1}\right)_{j} \rightarrow M\left(C_{1}\right)_{j+1}
$$

induced by $L_{1}$. By abuse of notation, $K_{j}$ is also the kernel of the map

$$
M\left(C_{2}\right)_{j} \rightarrow M\left(C_{2}\right)_{j+1} .
$$

From the exact sequence

$$
\begin{aligned}
0 \rightarrow H^{0}\left(\mathbf{P}^{n}, \mathscr{I}_{C_{1}}(t)\right) & \rightarrow H^{0}\left(\mathbf{P}^{n}, \mathscr{I}_{C_{1}}(t+1)\right) \\
& \rightarrow H^{0}\left(H_{1}, \mathscr{I}_{C_{1} \cap H_{1}}(t+1)\right) \rightarrow M\left(C_{1}\right)_{t} \rightarrow M\left(C_{1}\right)_{t+1} \\
\searrow & \nearrow \\
0 & \nearrow_{t} \nearrow_{t}
\end{aligned}
$$

and the analogous one for $C_{2}$, our hypothesis that $V_{1} \cap \Lambda\left(=C_{1} \cap H_{1}\right)$ and $V_{2} \cap \Lambda\left(=C_{2} \cap H_{1}\right)$ have the same Hilbert function implies that $C_{1}$ and $C_{2}$ have the same Hilbert function. Proceeding inductively, we get that $V_{1}$ and $V_{2}$ have the same Hilbert function, so we can apply Proposition 3.1.

Since the numerical character of a two-codimensional subscheme of $\mathbf{P}^{n}$ is defiined to be that of its intersection with a generic 2-plane (see [B-MR]), Corollary 3.6 says that if two subschemes are evenly linked and in the same shift and have the same numerical character then they lie in the same component of the Hilbert scheme.

Corollary 3.7. Fix an even liaison class $\mathscr{L}$ of two-codimensional subvarieties of $\mathbf{P}^{n}$. Those elements of $\mathscr{L}$ with fixed cohomology (i.e., with assigned dimensions $\left.h^{i}\left(\mathbf{P}^{n}, \mathscr{I}_{X}(t)\right), 0 \leq i \leq n-2, t \in \mathbf{Z}\right)$ are in an irreducible family.

Proof. If $\mathscr{L}$ is not arithmetically Cohen-Macaulay, then this follows from Proposition 3.1, since equalities for $i \geq 1$ imply that these subvarieties are in the same shift of $\mathscr{L}$. If $\mathscr{L}$ is arithmetically Cohen-Macaulay, the result is known [E, PS, B-MR].

Wiith this background we turn to the LR-problem introduced in $\S 1$. Our idea is to show that the approach introduced in [BM2] for curves in $\mathbf{P}^{3}$ can be 
extended to codimension 2 subschemes of $\mathbf{P}^{n}$. Notice that now a basic double link of a scheme $V$ depends only on one $F \in I_{V}$ and a general form $A \in S_{d}$. The first step (Corollary 3.9) is to show that without loss of generality we can restrict ourselves to basic double links using linear forms (i.e., $d=1$ ).

Lemma 3.8. Let $V_{1}$ and $V_{2}$ be two-codimensional subschemes of $\mathbf{P}^{n}$ with the same postulation and in the same shift of the same even liaison class. Let $F_{1}, G_{1} \in I_{V_{1}}$ and $F_{2}, G_{2} \in I_{V_{2}}$ such that $\operatorname{deg}\left(F_{1}\right)=\operatorname{deg}\left(F_{2}\right), \operatorname{deg}\left(G_{1}\right)=\operatorname{deg}\left(G_{2}\right)$, and both $I_{X_{1}}:=\left(F_{1}, G_{1}\right)$ and $I_{X_{2}}:=\left(F_{2}, G_{2}\right)$ are complete intersections. Let $V_{i} \sim_{X_{i}} W_{i}(i=1,2)$. Then $W_{1}$ and $W_{2}$ are in the same shift of the same even liaison class and have the same postulation.

Proof. Certainly $W_{1}$ and $W_{2}$ are in the same shift of the same even liaison class (namely the residual even liaison class), by Theorem 1.1. By the argument in Proposition 3.1 we get exact sequences

$$
\begin{aligned}
& 0 \rightarrow F \stackrel{u}{\rightarrow} \bigoplus_{1}^{m} \mathscr{O}_{\mathbf{P}^{n}}\left(-h_{i}\right) \rightarrow \mathscr{I}_{V_{1}} \rightarrow 0, \\
& 0 \rightarrow F \stackrel{u}{\rightarrow} \bigoplus_{1}^{m} \mathscr{O}_{\mathbf{P}^{n}}\left(-h_{i}\right) \rightarrow \mathscr{I}_{V_{2}} \rightarrow 0,
\end{aligned}
$$

where $H^{1}\left(\mathbf{P}^{n}, F(t)\right)=0$ for all $t$. It follows that

$$
h^{i}\left(\mathbf{P}^{n}, \mathscr{J}_{V_{1}}(t)\right)=h^{i}\left(\mathbf{P}^{n}, \mathscr{J}_{V_{2}}(t)\right)
$$

for $0 \leq i \leq n-1$ and for all $t$. Then from the exact sequence

$$
0 \rightarrow \mathscr{I}_{V_{i}} \rightarrow \mathscr{O}_{\mathbf{P}^{n}} \rightarrow \mathscr{O}_{V_{i}} \rightarrow 0
$$

we get $h^{n-2}\left(V_{1}, \mathscr{O}_{V_{1}}(t)\right)=h^{n-2}\left(V_{2}, \mathscr{O}_{V_{2}}(t)\right)$ for all $t$. By Serre duality, then, $h^{0}\left(V_{1}, \omega_{V_{1}}(t)\right)=h^{0}\left(V_{2}, \omega_{V_{2}}(t)\right)$ for all $t$. Since $h^{0}\left(\mathbf{P}^{n}, \mathscr{I}_{V_{1}}(t)\right)=h^{0}\left(\mathbf{P}^{n}, \mathscr{I}_{V_{2}}(t)\right)$ for all $t$, we get from the exact sequence

$$
0 \rightarrow \mathscr{I}_{X_{i}} \rightarrow \mathscr{I}_{W_{i}} \rightarrow \omega_{V_{i}} \rightarrow 0
$$

that $h^{0}\left(\mathbf{P}^{n}, \mathscr{J}_{W_{1}}(t)\right)=h^{0}\left(\mathbf{P}^{n}, \mathscr{J}_{W_{2}^{\prime}}(t)\right)$ for all $t$.

Corollary 3.9. (a) Let $V_{1}, V_{2}$ be two-codimensional subschemes of $\mathbf{P}^{n}$ with the same postulation and in the same shift of the same even liaison class. Let $F_{i} \in I_{V_{i}}$ where $F_{1}, F_{2}$ have the same degree, and let $A_{1}, A_{2} \in S_{d}$ (chosen generically). Suppose $W_{i}$ is a subscheme obtained from $V_{i}$ by a basic double linkage using $F_{i}$ and $A_{i}(i=1,2)$. Then $W_{1}$ and $W_{2}$ are in the same shift of the same even liaison class and have the same postulation.

(b) Let $V$ be a two-codimensional subscheme of $\mathbf{P}^{n}$ and let $F \in I_{V}$. Let $W_{1}$ be a basic double linkage obtained from $V$ using $F$ and a general form $A$ of degree $d$. Let $W_{2}$ be the result of a sequence of basic double linkages $V=V_{0}, V_{1}, \ldots, V_{d}=W_{2}$ such that each $V_{i}(1 \leq i \leq d)$ is obtained from $V_{i-1}$ 
using the same $F$ and a general linear form $L_{i}$. Then $W_{1}$ and $W_{2}$ are in the same shift of the same even liaison class and have the same postulation.

Proof. (a) follows from Lemma 3.8 and the definition of basic double linkage. The proof of (b) is identical to that in Lemma 1.6(b) of [BM2]. The main idea is that $W_{2}$ is really obtained by a single basic double linkage using $F \in I_{V}$ and the form $L_{1} L_{2} \cdots L_{d} \in S_{d}$, so we can apply (a).

The main idea of the preceding discussion is that the LR-property can be deduced from a study of the possible postulations within a shift of an even liaison class. The next theorem is our main tool in this regard and will be used in $\S 4$.

Theorem 3.10. Let $\mathscr{L}$ be an even liaison class of two-codimensional subschemes of $\mathbf{P}^{n}$. Assume that

(1) Any two subschemes $V_{1}, V_{2} \in \mathscr{L}^{0}$ have the same postulation, and

(2) For every subscheme $V \in \mathscr{L}^{h}(h>0)$ there exists a subscheme $W \in$ $\mathscr{L}^{h}$ with the same postulation as that of $V$ (possibly $V$ itself), satisfying the following condition:

there exist forms of some degrees $a, b$ linking $W$ to some subscheme $W_{1}$, and forms of degrees $a$ and $b-1$ linking $W_{1}$ to some $W_{2}$ (which is necessarily then in $\mathscr{L}^{h-1}$ ).

Then $\mathscr{L}$ has the LR-property.

Proof. By Proposition 3.1, assumption (1) implies condition (a) of the LRproperty (see Definition 1.8). So we have to show that (2) implies condition (b) of Definition 1.8. More precisely, we have to show that given $V \in \mathscr{L}^{h}(h>0)$ and any minimal $V_{0} \in \mathscr{L}^{0}$ there exists a sequence of subschemes $V_{0}, V_{1}, \ldots, V_{h}$ such that for each $i, V_{i} \in \mathscr{L}^{i}$ is a basic double link of $V_{i-1} \in \mathscr{L}^{i-1}$, and $V_{h}$ has the same postulation as that of $V$. The proof is by induction on $h$.

Let $V_{0} \in \mathscr{L}^{0}, V \in \mathscr{L}^{1}$. Let $W, W_{2}$ be as in the statement of (2) in the theorem. By (1), $V_{0}, W_{2} \in \mathscr{L}^{0}$ have the same postulation. Perform a basic double link on $V_{0}$ using a form $F \in I_{V_{0}}$ of degree $a$ and a linear form $L$, producing $V_{1} \in \mathscr{L}^{1} . \quad\left(F\right.$ exists since $h^{0}\left(\mathbf{P}^{n}, \mathscr{I}_{V_{0}}(a)\right)=h^{0}\left(\mathbf{P}^{n}, \mathscr{I}_{W_{2}}(a)\right) \neq 0$. $)$ By the way basic double linkage is defined (see Definition 1.7), it follows from Lemma 3.8 that $V_{1}$ and $W$ have the same postulation, so similarly for $V_{1}$ and $V$. Then apply Proposition 3.1.

The proof of the inductive step $(h>1)$ is identical to that just given for $h=1$, except of course that now $W_{2} \in \mathscr{L}^{h-1}$ is assumed by induction to have the same postulation as a "basic double link scheme" $V_{h-1} \in \mathscr{L}^{h-1}$.

Sometimes it will be of interest, conversely, to describe properties of even liaison classes which have the LR-property (conjecturally all even liaison classes, at least in codimension 2). Since our main computational tool is the study of 
postulations, it is important to know that this not only implies the LR-property but is in fact equivalent.

Proposition 3.11. Let $\mathscr{L}$ be an even liaison class in codimension 2. The following are equivalent:

(a) $\mathscr{L}$ has the LR-property.

(b) $\mathscr{L}$ satisfies

(i) Any two subschemes $V_{1}, V_{2} \in \mathscr{L}^{0}$ have the same postulation.

(ii) Given $V_{0} \in \mathscr{L}^{0}$ and $V \in \mathscr{L}^{h}(h \geq 1)$, there exists a sequence of subschemes $V_{0}, V_{1}, \ldots, V_{h}$ such that for all $i(1 \leq i \leq h), V_{i} \in \mathscr{L}^{i}$ is a basic double link of $V_{i-1}$ (using a linear form), and $V_{h}$ has the same postulation as that of $V$.

Proof. We have seen that (b) implies (a). Conversely, suppose that $\mathscr{L}$ has the LR-property. It is enough to show the following: if $\left\{X_{s}\right\}_{s \in S}$ is an irreducible flat family of two-codimensional subschemes of $\mathbf{P}^{n}$ such that $X_{s} \in \mathscr{L}^{h}$ for all $s \in$ $S$, then for any $t \in \mathbf{Z}$ and $a, b \in S$ we have $h^{0}\left(\mathbf{P}^{n}, \mathscr{J}_{X_{a}}(t)\right)=h^{0}\left(\mathbf{P}^{3}, \mathcal{I}_{X_{b}}(t)\right)$ (i.e., $X_{a}$ and $X_{b}$ have the same postulation).

We proceed by induction on $n \geq 3$. For $n=3$, the $X_{s}$ are curves in $\mathbf{P}^{3}$. Then the number $h^{0}\left(\mathbf{P}^{3}, \mathscr{I}_{X_{s}}(t)\right)-h^{1}\left(\mathbf{P}^{3}, \mathscr{J}_{X_{s}}(t)\right)+h^{2}\left(\mathbf{P}^{3}, \mathscr{I}_{X_{s}}(t)\right)$ is invariant throughout the family. But by hypothesis $h^{1}\left(\mathbf{P}^{3}, \mathscr{J}_{X_{s}}(t)\right)$ is also invariant, so we have that $h^{0}\left(\mathbf{P}^{3}, \mathscr{J}_{X_{s}}(t)\right)+h^{2}\left(\mathbf{P}^{3}, \mathscr{J}_{X_{s}}(t)\right)$ is invariant. Then the result follows from semicontinuity.

In $\mathbf{P}^{n}$ for $n \geq 4$, we let $H$ be a general hyperplane. By Lemma 3.5, linkage is preserved under proper hyperplane sections. Hence there is an irreducible family of two-codimensional subschemes of $H=\mathbf{P}^{n-1}$ containing $X_{a} \cap H$ and $X_{b} \cap H$, all the elements of which are in the same shift of the same even liaison class. By induction, for all $t$ we have $h^{0}\left(\mathbf{P}^{3}, \mathscr{I}_{X_{a} \cap H}(t)\right)=h^{0}\left(\mathbf{P}^{3}, \mathscr{I}_{X_{b} \cap H}(t)\right)$. The result then follows as in Corollary 3.4.

\section{AN EXAMPLE}

Let $Y$ be a two-codimensional subscheme of $\mathbf{P}^{n}$ with only one module $\left(M^{i}\right)(Y)$ nonzero, and that module concentrated in one degree with dimension $p>0$. We shall be interested in the even liaison class of $Y$, and in particular we shall show that it satisfies the LR-property.

The first observation to make is that up to shift, the cohomology just described determines the even liaison class of $Y$. Indeed, a repeated use of the exact sequence

$$
0 \rightarrow \mathscr{J}_{Y}(-1) \rightarrow \mathscr{I}_{Y} \rightarrow \mathscr{I}_{Y \cap H} \rightarrow 0
$$

for repeated hyperplane sections shows that if $Z$ is the intersection of $Y$ with a general linear subspace of dimension $m(3 \leq m \leq n-1)$ then $\left(M^{j}\right)(Z)$ has at most one nonzero component for each $j(1 \leq j \leq m-2)$. It follows 
trivially that each $\left(M^{j}\right)(Z)$ is annihilated by the maximal ideal $\boldsymbol{m}$ of $S$. Then by [SV] $Y$ is arithmetically Buchsbaum. But among arithmetically Buchsbaum two-codimensional subschemes of $\mathbf{P}^{n}$, the dimensions of the components of the modules $\left(M^{i}\right)$ (up to shift) completely determine the liaison class (use [C], then $[R])$.

Our proof will follow the inductive approach described in $\S 3$, especially Theorem 3.10. The techniques for forcing the existence of "good" linkages, which we use frequently in this section, were first used in [BM1]. It is thus necessary to consider the possible shifts of the associated module which can actually occur in the even liaison class.

Definition 4.1. Fix a positive integer $p$. Then for any $n \geq 3$ and $1 \leq i \leq$ $n-2, \mathscr{L}_{n, i}$ is the set of two-codimensional subschemes $Y$ of $\mathbf{P}^{n}$ having $h^{i}\left(\mathbf{P}^{n}, \mathscr{J}_{Y}(t)\right)=p$ for some $t$, and $h^{j}\left(\mathbf{P}^{n}, \mathscr{I}_{Y}(s)\right)=0$ for all $1 \leq j \leq n-2$ and $(j, s) \neq(i, t)$. Note that $\mathscr{L}_{n, i}$ is an even liaison class, and the residual even liaison class is $\mathscr{L}_{n, n-i-1}$.

Definition 4.2. (i) If $Y \subset \mathbf{P}^{n}$ is a subscheme then

$$
\alpha(Y):=\min \left\{\operatorname{deg} F \mid F \in I_{Y}\right\} .
$$

(ii) If $\mathscr{L}$ is an even liaison class in $\mathbf{P}^{n}$ then

$$
\alpha(\mathscr{L}):=\min \left\{\operatorname{deg} F \mid F \in I_{Y} \text { for some } Y \in \mathscr{L}\right\} .
$$

Note that for any even liaison class $\mathscr{L}$, we have $\alpha(Y) \geq \alpha(\mathscr{L})$ for all $Y \in \mathscr{L}$. Also, since $\alpha(\mathscr{L})=\alpha\left(\mathscr{L}^{\vee}\right)$ (the residual even liaison class) we have $\alpha\left(\mathscr{L}_{n, i}\right)=\alpha\left(\mathscr{L}_{n, n-i-1}\right)$. However, we shall see that in general we do not have $\alpha\left(\mathscr{L}_{n, i}\right)=\alpha\left(\mathscr{L}_{n, j}\right)$ for $i \neq j$ (apart from $\left.j=n-i-1\right)$. In fact (Corollary $4.10(\mathrm{a}))$ we have $a\left(\mathscr{L}_{n, i}\right)=\left(\begin{array}{c}n-1 \\ i\end{array}\right) p$. This partially generalizes a result of Amasaki [A, GM2] for arithmetically Buchsbaum curves in $\mathbf{P}^{3}$. A stronger generalization, which includes our Corollary $4.10(\mathrm{a})$, is contained in the recent work [C]. However, we believe that the techniques used in this section should be applicable to non-Buchsbaum subschemes of $\mathbf{P}^{n}$ as well, so we will not take the slight shortcut provided by [C].

Let $Y \in \mathscr{L}_{n, i}$. Let $H_{1}, \ldots, H_{n-2}$ be general hyperplanes and for any $j$ $(1 \leq j \leq n-2)$ let $W_{j}=H_{1} \cap \cdots \cap H_{j}=\mathbf{P}^{n-j}$. Let $Y_{0}=Y$ and $Y_{j}=Y \cap W_{j}$. Note that $\operatorname{dim} Y_{j}=n-2-j$ and $Y_{j} \subset W_{j}=\mathbf{P}^{n-j}$.

Lemma 4.3. Let $Y \in \mathscr{L}_{n, i}$ and assume that $h^{i}\left(\mathbf{P}^{n}, \mathscr{I}_{Y}(k)\right)=p$. Let $Y_{j}$ be as above. Then

(a) $Y_{n-3} \in \mathscr{L}_{3, N}$ where $N=\left(\begin{array}{c}n-3 \\ i-1\end{array}\right) p$. In fact, $h^{1}\left(\mathbf{P}^{3}, \mathscr{J}_{Y_{n-3}}(k+i-1)\right)=N$.

(b) $k \geq \alpha(Y)-i-1$. 
Proof. By repeatedly using the exact sequence $(*)$ at the beginning of this section (see also [B-MR]) we get

$$
\begin{aligned}
h^{1}\left(\mathbf{P}^{3},\right. & \left.\mathscr{I}_{Y_{n-3}}(k+i-1)\right) \\
= & h^{1}\left(\mathbf{P}^{4}, \mathscr{J}_{Y_{n-4}}(k+i-1)\right)+h^{2}\left(\mathbf{P}^{4}, \mathscr{I}_{Y_{n-4}}(k+i-2)\right) \\
= & h^{1}\left(\mathbf{P}^{5}, \mathscr{J}_{Y_{n-5}}(k+i-1)\right)+2 h^{2}\left(\mathbf{P}^{5}, \mathscr{I}_{Y_{n-5}}(k+i-2)\right) \\
& +h^{1}\left(\mathbf{P}^{5}, \mathscr{J}_{Y_{n-5}}(k+i-3)\right) \\
= & h^{1}\left(\mathbf{P}^{6}, \mathscr{J}_{Y_{n-6}}(k+i-1)\right)+3 h^{2}\left(\mathbf{P}^{6}, \mathscr{I}_{Y_{n-6}}(k+i-2)\right) \\
& +3 h^{3}\left(\mathbf{P}^{6}, \mathscr{I}_{Y_{n-6}}(k+i-3)\right)+h^{4}\left(\mathbf{P}^{6}, \mathscr{I}_{Y_{n-6}}(k+i-4)\right) \\
& \quad \vdots \\
= & h^{1}\left(\mathbf{P}^{n}, \mathscr{I}_{Y}(k+i-1)\right)+\left(\begin{array}{c}
n-3 \\
1
\end{array}\right) h^{2}\left(\mathbf{P}^{n}, \mathscr{I}_{Y}(k+i-2)\right)+\cdots \\
& +\left(\begin{array}{c}
n-3 \\
i-1
\end{array}\right) h^{i}\left(\mathbf{P}^{n}, \mathscr{I}_{Y}(k)\right)+\cdots+h^{n-2}\left(\mathbf{P}^{n}, \mathscr{I}_{Y}(k+i-n+2)\right) \\
= & \left(\begin{array}{c}
n-3 \\
i-1
\end{array}\right) p .
\end{aligned}
$$

This proves (a). Now, exactly as in [GM1, Corollary 3.9], at any stage we have $\alpha\left(Y_{j}\right)-1 \leq \alpha\left(Y_{j+1}\right) \leq \alpha\left(Y_{j}\right)$. Furthermore, $\alpha\left(Y_{j}\right)-1=\alpha\left(Y_{j+1}\right)$ if and only if $h^{1}\left(\mathbf{P}^{n-j}, \mathscr{I}_{Y_{j}}\left(\alpha\left(Y_{j}\right)-2\right)\right) \neq 0$. Since $k+i-1$ is the only degree in which there can possibly be a nonzero $H^{1}$, for any $Y_{j}$, the phenomenon $\alpha\left(Y_{j}\right)-1=\alpha\left(Y_{j+1}\right)$ can happen at most once during our sequence of hyperplane sections. Thus, $\alpha(Y)-1 \leq \alpha\left(Y_{n-3}\right) \leq \alpha(Y)$.

We consider two cases. If $\alpha(Y)-1=\alpha\left(Y_{n-3}\right)$, then $\alpha(Y)-2=k+i-1$ and we are done. If $\alpha\left(Y_{n-3}\right)=\alpha(Y)$, then by [GM1, Corollary 3.9], $k+i-1 \geq$ $\alpha(Y)-2$ and again we are done.

Lemma 4.3 (b) gives us a lower bound for the possible shifts of $\mathscr{L}_{n, i}$ that can occur in terms of $\alpha\left(\mathscr{L}_{n, i}\right)$. It can be shown using [C] that this is sharp (although a simpler reason will emerge in Lemma 4.7(a)). We now use this to rewrite Definition 1.5 in a precise way for our present situation.

Definition 4.4. Let $\alpha=\alpha\left(\mathscr{L}_{n, i}\right)$. We write $Y \in \mathscr{L}_{n, i}^{h}(h \geq 0)$ if and only if $Y \in \mathscr{L}_{n, i}$ and $h^{i}\left(\mathbf{P}^{n}, \mathscr{I}_{Y}(\alpha-i-1+h)\right)=p$.

Note that if $Y \in \mathscr{L}_{n, i}^{0}$ then $Y$ must lie on a hypersurface of degree $\alpha$ by Lemma 4.3. Now, as usual, for a two-codimensional $Y \subset \mathbf{P}^{n}$ we define

$$
e(Y)=\max \left\{t \mid h^{n-1}\left(\mathbf{P}^{n}, \mathscr{I}_{Y}(t)\right) \neq 0\right\} .
$$

Lemma 4.5. Let $\alpha=\alpha\left(\mathscr{L}_{n, i}\right)$. Let $Y \in \mathscr{L}_{n, i}^{h}$ and assume that $Y$ lies on $a$ hypersurface of degree $\alpha$. Then $e(Y) \leq \alpha-n-1+h$. 
Proof. Suppose otherwise, i.e., that $e(Y) \geq \alpha-n+h$. Consider the following table for the cohomology of $\mathscr{I}_{Y}$ :

\begin{tabular}{cccccccccc}
$\mathscr{I}_{Y}$ & $\alpha-1$ & $\alpha$ & $\cdots$ & $\alpha-n+h$ & $\alpha-n+1+h$ & $\cdots$ & $\alpha-i-1+h$ & $\alpha-i+h$ & $\cdots$ \\
\hline$h^{0}$ & 0 & $*$ & $\cdots$ & $*$ & $*$ & $\cdots$ & $*$ & $*$ & $\cdots$ \\
$h^{1}$ & 0 & 0 & $\cdots$ & 0 & 0 & $\cdots$ & 0 & 0 & $\cdots$ \\
$\vdots$ & & & $\cdots$ & & & $\cdots$ & & & $\cdots$ \\
$h^{i}$ & 0 & 0 & $\cdots$ & 0 & 0 & $\cdots$ & $p$ & 0 & $\cdots$ \\
$\vdots$ & & & $\cdots$ & & & $\cdots$ & & & $\cdots$ \\
$h^{n-2}$ & 0 & 0 & $\cdots$ & 0 & 0 & $\cdots$ & 0 & 0 & $\cdots$ \\
$h^{n-1}$ & & & & $*$ & $?$ & & & &
\end{tabular}

Since $e \geq \alpha-n+h$ we have

$$
\begin{aligned}
0 & =h^{n-1}\left(\mathbf{P}^{n}, \mathscr{I}_{Y}(e+1)\right)=h^{n-2}\left(\mathbf{P}^{n}, \mathscr{I}_{Y}(e+2)\right) \\
& =\cdots=h^{i}\left(\mathbf{P}^{n}, \mathscr{I}_{Y}(e+n-i)\right)=\cdots=h^{1}\left(\mathbf{P}^{n}, \mathscr{I}_{Y}(e+n-1)\right) .
\end{aligned}
$$

By Castelnuovo-Mumford, then, $\mathscr{I}_{Y}$ is globally generated in degree $e+n$. Hence we can perform a link using a hypersurface of degree $\alpha$ and one of degree $e+n$, to a residual $Y^{\prime} \in \mathscr{L}_{n, n-i-1}$. Let $X$ be the complete intersection. Then by [PS] we have an exact sequence

$$
0 \rightarrow \mathscr{I}_{X}(\alpha-1) \rightarrow \mathscr{I}_{Y^{\prime}}(\alpha-1) \rightarrow \omega_{Y}(-e) \rightarrow 0 .
$$

But by the definition of $e$ and standard isomorphisms, we know $h^{0}\left(Y, \omega_{Y}(-e)\right)$ $\neq 0$. Therefore since $h^{1}\left(\mathbf{P}^{n}, \mathscr{J}_{X}(\alpha-1)\right)=0$ we have $h^{0}\left(\mathbf{P}^{n}, \mathscr{I}_{Y^{\prime}}(\alpha-1)\right) \neq 0$. But $\alpha=\alpha\left(\mathscr{L}_{n, i}\right)=\alpha\left(\mathscr{L}_{n, n-i-1}\right)$. Impossible.

Lemma 4.6. Let $Y \in \mathscr{L}_{n, i}$ and set $s=\alpha(Y)$. Assume that

$$
h^{i}\left(\mathbf{P}^{n}, \mathscr{J}_{Y}(s-i-1)\right)=p \text {. }
$$

Then

$h^{0}\left(\mathbf{P}^{n}, \mathscr{J}_{Y}(s)\right) \geq p\left[\left(\begin{array}{l}n-2 \\ n-2\end{array}\right)+\left(\begin{array}{l}n-2 \\ n-3\end{array}\right)+\cdots+\left(\begin{array}{c}n-2 \\ i+1\end{array}\right)\right]+2\left(\begin{array}{c}n-2 \\ i\end{array}\right) p+1$.

Proof. This is a continuation of the reasoning in Lemma 4.3, and we continue to use the notation introduced there. We consider two cases (since we can get a stronger result for $i=n-2$ ).

If $i=n-2$ then

$$
h^{0}\left(\mathbf{P}^{n}, \mathscr{I}_{Y}(s)\right)=h^{0}\left(\mathbf{P}^{n-1}, \mathscr{I}_{Y_{1}}(s)\right)=\cdots=h^{0}\left(\mathbf{P}^{3}, \mathscr{I}_{Y_{n-3}}(s)\right) \text {. }
$$

But $k=s-i-1$ and $h^{1}\left(\mathbf{P}^{3}, \mathscr{J}_{Y_{n-3}}(k+i-1)\right)=p$ (Lemma 4.3); i.e., $h^{1}\left(\mathbf{P}^{3}, \mathscr{I}_{Y_{n-3}}(s-2)\right)=p$. Then from [BM2, Lemma 2.15] we have

$$
h^{0}\left(\mathbf{P}^{n}, \mathscr{J}_{Y}(s)\right)=h^{0}\left(\mathbf{P}^{3}, \mathscr{I}_{Y_{n-3}}(s)\right) \geq 3 p+1 .
$$

If $1 \leq i \leq n-3$ then $H_{*}^{1}\left(\mathbf{P}^{n-j}, \mathscr{I}_{Y_{j}}\right)=0$ for $j \leq i-2$ and

$$
h^{1}\left(\mathbf{P}^{n-i+1}, \mathscr{J}_{Y_{l-1}}(s-2)\right)=p .
$$


Note also that $h^{1}\left(\mathbf{P}^{n-j}, \mathscr{J}_{Y_{j}}(s-1)\right)=0$ for all $1 \leq j \leq n-3$. Then from the exact sequences

$$
\begin{aligned}
0 \rightarrow H^{0}\left(\mathbf{P}^{n-j+1},\right. & \left.\mathscr{J}_{Y_{j-1}}(s-2)\right) \rightarrow H^{0}\left(\mathbf{P}^{n-j+1}, \mathscr{I}_{Y_{j-1}}(s-1)\right) \\
& \rightarrow H^{0}\left(\mathbf{P}^{n-j}, \mathscr{J}_{Y_{j}}(s-1)\right) \rightarrow H^{1}\left(\mathbf{P}^{n-j+1}, \mathscr{I}_{Y_{j-1}}(s-2)\right) \rightarrow 0
\end{aligned}
$$

and

$$
\begin{aligned}
& 0 \rightarrow H^{0}\left(\mathbf{P}^{n-j+1}\right.\left., \mathscr{I}_{Y_{j-1}}(s-1)\right) \rightarrow H^{0}\left(\mathbf{P}^{n-j+1}, \mathscr{I}_{Y_{j-1}}(s)\right) \\
& \rightarrow H^{0}\left(\mathbf{P}^{n-j}, \mathscr{J}_{Y_{j}}(s)\right) \rightarrow 0
\end{aligned}
$$

we get in particular $h^{0}\left(\mathbf{P}^{n-i}, \mathscr{I}_{Y_{i}}(s-1)\right)=p$. Continuing to take hyperplane sections and using the same reasoning as in Lemma 4.3, we get

$$
h^{0}\left(\mathbf{P}^{n-j}, \mathscr{J}_{Y_{j}}(s-1)\right)=\left(\begin{array}{c}
n-2 \\
i-j+n-2
\end{array}\right) p \quad(i \leq j \leq n-2) .
$$

Now, $Y_{n-2}$ is a subscheme of $\mathbf{P}^{2}$, so we may apply [GM1, Proposition 4.2] to get $h^{0}\left(\mathbf{P}^{2}, \mathscr{J}_{Y_{n-2}}(s)\right) \geq 2\left(\begin{array}{c}n-2 \\ i\end{array}\right) p+1$. Then

$$
\begin{aligned}
h^{0}\left(\mathbf{P}^{n}, \mathscr{J}_{Y}(s)\right)= & h^{0}\left(\mathbf{P}^{n-1}, \mathscr{I}_{Y_{1}}(s)\right) \\
& \vdots \\
= & h^{0}\left(\mathbf{P}^{n-i}, \mathscr{I}_{Y_{i}}(s)\right) \\
= & p+h^{0}\left(\mathbf{P}^{n-i-1}, \mathscr{J}_{Y_{i+1}}(s)\right) \\
& \vdots \\
= & p\left[\left(\begin{array}{c}
n-2 \\
n-2
\end{array}\right)+\left(\begin{array}{c}
n-2 \\
n-3
\end{array}\right)+\cdots+\left(\begin{array}{c}
n-2 \\
i+1
\end{array}\right)\right]+h^{0}\left(\mathbf{P}^{2}, \mathscr{I}_{Y_{n-2}}(s)\right) \\
\geq & p\left[\left(\begin{array}{c}
n-2 \\
n-2
\end{array}\right)+\left(\begin{array}{c}
n-2 \\
n-3
\end{array}\right)+\cdots+\left(\begin{array}{c}
n-2 \\
i+1
\end{array}\right)\right] \\
& +2\left(\begin{array}{c}
n-2 \\
i
\end{array}\right) p+1 .
\end{aligned}
$$

Lemma 4.7. Let $\alpha=\alpha\left(\mathscr{L}_{n, i}\right)$. Let $Y \in \mathscr{L}_{n, i}^{h}$ and assume that $Y$ lies on a hypersurface of degree $\alpha$. Then

(a) $Y$ is directly linked to a subscheme $Y^{\prime} \in \mathscr{L}_{n, n-i-1}^{0}$;

(b) $e(Y)=\alpha-n-1+h$.

Proof. The cohomology of $\mathscr{I}_{Y}$ is similar to that in the table in the proof of Lemma 4.5, except that now we know $e(Y) \leq \alpha-n-1+h$. Hence, again 
invoking Castelnuovo-Mumford, we get a link using hypersurfaces of degree $\alpha$ and $\alpha+h$ to $Y^{\prime}$. A computation using Theorem 1.1 gives (a).

For (b), we use the exact sequence

$$
0 \rightarrow \mathscr{I}_{X}(\alpha) \rightarrow \mathscr{I}_{Y^{\prime}}(\alpha) \rightarrow \omega_{Y}(n+1-\alpha-h) \rightarrow 0
$$

(where again $X$ is the complete intersection). Then by Lemma 4.6 we get $e(Y) \geq \alpha-n-1+h$ and so we are done.

Now, as in the proof of the LR-property for arithmetically Buchsbaum curves in $\mathbf{P}^{3}$ [BM2], we have one special case which is not easily handled in our inductive argument. Fortunately, however, these subschemes are easily described (Proposition 4.9).

Lemma 4.8. Let $\alpha=\alpha\left(\mathscr{L}_{n, i}\right)$ and let $Y \in \mathscr{L}_{n, i}^{h}$. Then $e(Y) \geq \alpha-5+h$.

Proof. If $i=n-2$ we have $h^{n-2}\left(\mathbf{P}^{n}, \mathscr{J}_{Y}(\alpha+h-n+1)\right)=p$ and the result follows from [B-MR, Corollary 2.2]. If $1 \leq i<n-2$ the idea is simply to cut by enough hyperplanes until the result has its "top" $\left(M^{i}\right) \neq 0$; then use [B-MR]. That is, we cut by a sequence of $n-i-2$ general hyperplanes. One checks that

$$
\begin{aligned}
e(Y)= & e\left(Y_{1}\right)-1 \\
= & e\left(Y_{2}\right)-2 \\
& \vdots \\
= & e\left(Y_{n-i-2}\right)-(n-i-2) \\
\geq & (\alpha-i-1+h)-2-(n-i-2) \quad[\mathrm{B}-\mathrm{MR}] \\
= & \alpha-1-n+h .
\end{aligned}
$$

(See the paragraph preceding Lemma 4.3 for notation.)

The special case we must consider is when the bounds of Lemmas 4.3 and 4.8 are simultaneously attained.

Proposition 4.9. Let $\alpha=\alpha\left(\mathscr{L}_{n, i}\right)$. Let $Y \in \mathscr{L}_{n, i}^{h}$ with $s:=\alpha(Y)=\alpha+h$ and $e(Y)=\alpha-n-1+h$. Then the Hilbert function of $Y$ is completely determined, and

(a) $s=\left(\begin{array}{c}n-1 \\ i\end{array}\right) p$.

(b) $\operatorname{deg} Y=\left(\begin{array}{c}s \\ 2\end{array}\right)+\left(\begin{array}{c}n-2 \\ i\end{array}\right) p$ where $s=\left(\begin{array}{c}n-1 \\ i\end{array}\right) p$.

Proof. Note that $s=\alpha+h$ if and only if $h^{i}\left(\mathbf{P}^{n}, \mathscr{I}_{Y}(s-i-1)\right)=p$ (i.e., the bound of Lemma 4.3 is attained) since by Definition 4.4

$$
h^{i}\left(\mathbf{P}^{n}, \mathscr{F}_{Y}(\alpha-i-1+h)\right)=p .
$$


Now we have the cohomology table

\begin{tabular}{cccccccccc}
$\mathscr{I}_{Y}$ & $s-n-1$ & $s-n$ & $\cdots$ & $s-i-2$ & $s-i-1$ & $s-i$ & $\cdots$ & $s-1$ & $s$ \\
\hline$h^{0}$ & 0 & 0 & 0 & 0 & 0 & 0 & $*$ \\
$h^{1}$ & 0 & 0 & 0 & 0 & 0 & 0 & 0 \\
$\vdots$ & & & & & & & & & \\
$h^{i}$ & 0 & 0 & 0 & $p$ & 0 & 0 & 0 \\
$\vdots$ & & & & & & & & \\
$h^{n-2}$ & 0 & 0 & 0 & 0 & 0 & 0 & 0 \\
$h^{n-1}$ & $*$ & 0 & 0 & 0 & 0 & 0 & 0
\end{tabular}

By Castelnuovo-Mumford we can perform a link using two hypersurfaces of degree $s$ to $Y^{\prime} \in \mathscr{L}_{n, n-i-1}^{h}$.

From the exact sequence

$$
0 \rightarrow \mathscr{I}_{X}(s-1) \rightarrow \mathscr{I}_{Y^{\prime}}(s-1) \rightarrow \omega_{Y}(n-s) \rightarrow 0
$$

(where $X$ again is the complete intersection) plus the analogous one with $Y$ and $Y^{\prime}$ reversed, we note that $Y^{\prime}$ also satisfies the hypotheses in the statement of the proposition, but for $\mathscr{L}_{n, n-i-1}^{h}$.

Now we continue to use the notation $Y_{j}=Y \cap H_{1} \cap \cdots \cap H_{j}$. Because $h^{0}\left(\mathbf{P}^{n}, \mathscr{J}_{Y}(s-1)\right)=0$, the same argument as in Lemma 4.3 gives

$$
h^{0}\left(\mathbf{P}^{2}, \mathscr{J}_{Y_{n-2}}(s-1)\right)=\left(\begin{array}{c}
n-2 \\
i
\end{array}\right) p .
$$

Similarly, $h^{0}\left(\mathbf{P}^{2}, \mathcal{J}_{Y_{n-2}^{\prime}}(s-1)\right)=\left(\begin{array}{c}n-2 \\ n-i-1\end{array}\right) p$ and $\alpha\left(Y_{n-2}^{\prime}\right)=\alpha\left(Y_{n-2}\right)=s-1$.

Following the ideas of [GM1], we graph the function $\Delta H\left(Y_{n-2}, t\right)$ (the first difference function of the Hilbert function):

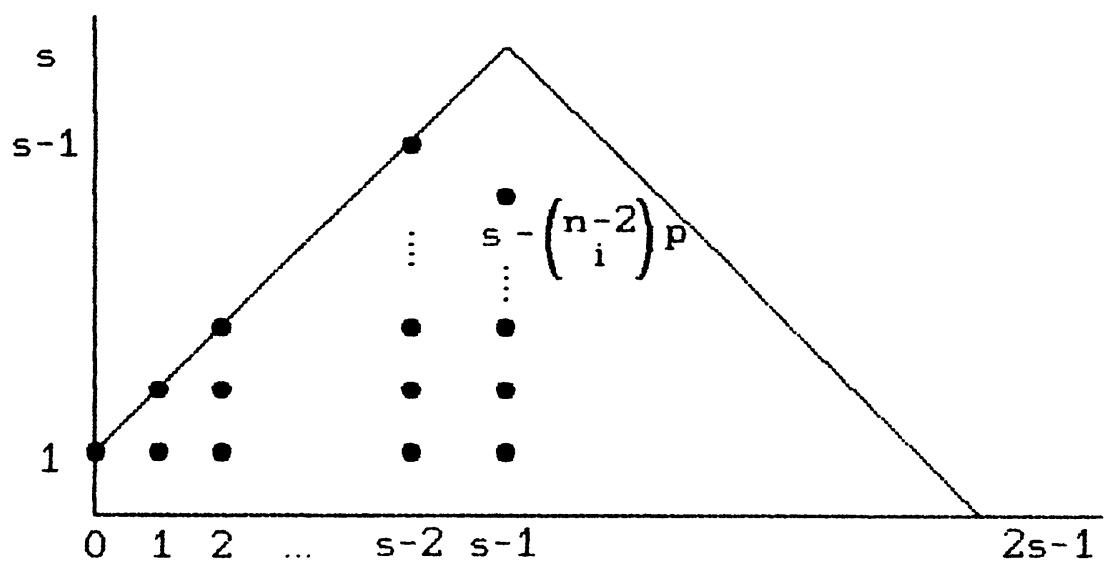

FIGURE 1 
The solid dots represent $\Delta H\left(Y_{n-2}, t\right)$, while the line represents the first difference of the Hilbert function of the complete intersection $X_{n-2}=X \cap H_{1} \cap$ $\cdots \cap H_{n-2}$ (i.e., $\left.\Delta H\left(X_{n-2}, t\right)\right)$.

At the moment we cannot go beyond degree $s-1$. However, recall that the graph of $\Delta H\left(Y_{n-2}^{\prime}, t\right)$ can be read from that of $Y_{n-2}$ by reading from right to left, under the graph of $\Delta H\left(X_{n-2}, t\right)$ and above that of $\Delta H\left(Y_{n-2}, t\right)$ [DGO]. Since $\alpha\left(Y_{n-2}^{\prime}\right)=s-1$ we get $\Delta H\left(Y_{n-2}, s\right)=0$ by a quick calculation. Thus the Hilbert function of $Y_{n-2}$ is uniquely determined, and so also that of $Y$ (as in Corollary 3.4 and Corollary 3.6).

Furthermore, as noted above, we have $h^{0}\left(\mathbf{P}^{2}, \mathscr{I}_{Y_{n-2}^{\prime}}(s-1)\right)=\left(\begin{array}{c}n-2 \\ n-i-1\end{array}\right) p$. On the other hand, from Figure 1 we get $h^{0}\left(\mathbf{P}^{2}, \mathscr{I}_{Y_{n-2}^{\prime}}(s-1)\right)=s-\left(\begin{array}{c}n-2 \\ i\end{array}\right) p$. Hence

$$
s=\left(\begin{array}{c}
n-2 \\
i
\end{array}\right) p+\left(\begin{array}{c}
n-2 \\
n-i-1
\end{array}\right) p=\left(\begin{array}{c}
n-1 \\
i
\end{array}\right) p .
$$

For (b), recall that $\operatorname{deg} Y=\operatorname{deg} Y_{n-2}=$ "integral" of the graph of $\Delta H\left(Y_{n-2}, t\right)$. ("Count the dots".) A calculation gives the result.

Corollary 4.10. (a) $\alpha\left(\mathscr{L}_{n, i}\right)=\left(\begin{array}{c}n-1 \\ i\end{array}\right) p$.

(b) If $Y \in \mathscr{L}_{n, i}^{h}$ lies on a hypersurface of degree $\left(\begin{array}{c}n-1 \\ i\end{array}\right) p$ then $e(Y)=\left(\begin{array}{c}n-1 \\ i\end{array}\right) p+$ $h-n-1$.

(c) The subschemes described in Proposition 4.9 occur if and only if $h=0$.

Proof. Let $\alpha=\alpha\left(\mathscr{L}_{n, i}\right)$. Let $Y \in \mathscr{L}_{n, i}^{h}$. If $h=0$ then by the observation following Definition 4.4 we have $\alpha(Y)=\alpha$, and by Lemma $4.7 e(Y)=\alpha-n-1$. Hence $Y$ satisfies the hypotheses of Proposition 4.9, and we have half of (c).

Now, merely assume that $Y \in \mathscr{L}_{n, i}^{h}$ lies on a hypersurface of degree $\alpha$. By Lemma 4.7, $Y$ is directly linked to $Y^{\prime} \in \mathscr{L}_{n, n-i-1}^{0}$. But we have just seen that $Y^{\prime}$ satisfies the hypotheses of Proposition 4.9, so $\alpha=\alpha(Y) \geq \alpha\left(Y^{\prime}\right)=$ $\left(\begin{array}{c}n-1 \\ n-i-1\end{array}\right) p=\left(\begin{array}{c}n-1 \\ i\end{array}\right) p$. but $\alpha\left(Y^{\prime}\right) \geq \alpha$ by definition of $\alpha$, so we are done (a).

Then (b) is immediate from Lemma 4.7, and the second half of (c) from the equality $s=\alpha+h$.

Example 4.11. Consider the case of surfaces in $\mathbf{P}^{4}$. We can produce a minimal surface $Y \in \mathscr{L}_{4,1}^{0}$ (for any $p$ ) by Liaison Addition, as follows. For $p=1$, let $Y_{1}$ be a general projection to $\mathbf{P}^{4}$ of the Veronese surface in $\mathbf{P}^{5}$ (see Example 1.6). So $\operatorname{deg} Y_{1}=4, \alpha\left(Y_{1}\right)=3, h^{0}\left(\mathbf{P}^{4}, \mathscr{J}_{Y_{1}}(3)\right)=7, h^{1}\left(\mathbf{P}^{4}, \mathscr{I}_{Y_{1}}(1)\right)=1$, $h^{1}\left(\mathbf{P}^{4}, \mathscr{J}_{Y_{1}}(t)\right)=0$ for $t \neq 1, H_{*}^{2}\left(\mathbf{P}^{4}, \mathscr{J}_{Y_{1}}\right)=0$. Applying Liaison Addition to $Y_{1}$ and another such surface using two cubic hypersurfaces gives $Y_{2}$ with $h^{1}\left(\mathbf{P}^{4}, \mathscr{J}_{Y_{2}}(4)\right)=2$, and continuing this $p$ times gives $Y=Y_{p} \in \mathscr{L}_{4,1}$. A simple calculation gives $h=0$, and one can directly verify the numerical results of Proposition 4.9.

Thanks to the recent paper of Chang [C], this procedure can in fact be used in more general situations. 
Theorem 4.12. $\mathscr{L}_{n, i}$ has the LR-property.

Proof. We follow the inductive approach described in $\S 3$, especially Theorem 3.10. For $h=0$ it follows from Proposition 4.9 and Corollary 4.10 that the postulation of a subscheme in $\mathscr{L}_{n, i}^{0}$ is uniquely determined. So let $h>0$. Let $Y \in \mathscr{L}_{n, i}^{h}$. Let $s=\alpha(Y)$ and $\alpha=\left(\begin{array}{c}n-1 \\ i\end{array}\right) p$.

If $e=e(Y) \geq \alpha-n+h$ then, as above, in either case we can perform a link using hypersurfaces of degree $s$ and $e+n$ to a subscheme $W_{1}$. Let $X$ be the complete intersection. From the exact sequence

$$
0 \rightarrow \mathscr{I}_{X}(s-1) \rightarrow \mathscr{I}_{W_{1}}(s-1) \rightarrow \omega_{Y}(-e) \rightarrow 0
$$

we get the existence of a link on $W_{1}$ using hypersurfaces of degree $s-1$ and $e+n$ to $W_{2}$ as in Theorem 3.10 .

If $e=e(Y)=\alpha-n-1+h$ and $s<\alpha+h$, we can perform a link using hypersurfaces of degree $s$ and $\alpha+h$ to $W_{1}$. We have

$$
0 \rightarrow \mathscr{I}_{X}(s-1) \rightarrow \mathscr{I}_{Y}(s-1) \rightarrow \omega_{W_{1}}(n-\alpha-h) \rightarrow 0
$$

so $e\left(W_{1}\right) \leq \alpha+h-n-1$. Now, if $Y \in \mathscr{L}_{n, i}^{h}$ we have $h^{n-i-1}\left(\mathbf{P}^{n}, \mathscr{J}_{W_{1}}(s+i-n)\right)=$ $p$ (Theorem 1.1), so $h^{n-i-1}\left(\mathbf{P}^{n}, \mathscr{J}_{W_{1}}(t)\right)=0$ for all $t \geq s+i-n+1$. But note that $s+i-n+1 \leq \alpha+h-n+i$. Thus

$$
\begin{aligned}
0= & h^{n-1}\left(\mathbf{P}^{n}, \mathscr{I}_{W_{1}}(\alpha+h-n)\right) \\
= & h^{n-2}\left(\mathbf{P}^{n}, \mathscr{I}_{W_{1}}(\alpha+h-n+1)\right) \\
& \vdots \\
= & h^{1}\left(\mathbf{P}^{n}, \mathscr{I}_{W_{1}}(\alpha+h-2)\right) .
\end{aligned}
$$

It follows from Castelnuovo-Mumford that we can link using hypersurfaces of degree $s$ and $\alpha+h-1$ to $W_{2}$ as in Theorem 3.10.

The only other possibility is that $e=e(Y)=\alpha+h-n-1$ and $s=\alpha+h$. But by Proposition 4.9 and Corollary 4.10(c) we have $h=0$ and $Y$ is minimal.

Example 4.13. It follows trivially from Theorem 4.12 that in the entire liaison class $\mathscr{L}$ of the general projection to $\mathbf{P}^{4}$ of the Veronese in $\mathbf{P}^{5}$ (see Example 1.6) there is no surface of degree 6 (smooth or otherwise). If $V \in \mathscr{L}$ and $\operatorname{deg} V=5$ then it is oddly linked to the Veronese and has sectional (arithmetic) genus 1. If $\operatorname{deg} V=7$ then it is evenly linked to the Veronese and has sectional genus 4. For each of degrees 8 and 9 there are two possibilities (one even and one odd) and so on. (See also [O2] for a classification of smooth surfaces in $\mathbf{P}^{4}$ of low degree up to liaison and [B-MR] for a discussion of when surfaces in $\mathscr{L}_{n}$ are smoothable.) This kind of problem will be studied again in the next section. 


\section{ABout BASIC DOUBLE LINKS}

In this section we discuss how to handle sequences of basic double links. We show that for an even liaison class $\mathscr{L}$ with the LR-property, there exists a "standard form" of sequences of basic double links for any given element of $\mathscr{L}$. This allows us to describe $\mathscr{L}$ as a union of disjoint nonvoid subsets, parameterized in a very simple way. We then give a reinterpretation of basic double linkages in terms of liaison addition. This provides, a fortiori, a new way of looking at the LR-property and in particular clarifies the naive idea that "a subscheme obtained by a sequence of basic double links from $X$ is like $X$ with an arithmetically Cohen-Macaulay tail attached."

First we need more precise notation for sequences of basic double links:

Definition 5.1. Let $X$ be a two-codimensional subscheme of $\mathbf{P}^{n}$. When we write that

$$
X=X_{0}:\left(b_{1}, f_{1}\right) \rightarrow X_{1}:\left(b_{2}, f_{2}\right) \rightarrow \cdots \rightarrow X_{k-1}:\left(b_{k}, f_{k}\right) \rightarrow X_{k}
$$

is a sequence of basic double links, we mean that each $X_{i}$ is obtained from $X_{i-1}$ via a basic double link with a hypersurface of degree $b_{i}$ containing $X_{i-1}$ and a general hypersurface of degree $f_{i}$.

The following lemma is the first step for substituting a given sequence of basic double links with a simpler one.

Lemma 5.2. Let

$$
X=X_{0}:\left(b_{1}, f_{1}\right) \rightarrow X_{1}:\left(b_{2}, f_{2}\right) \rightarrow \cdots \rightarrow X_{p-1}:\left(b_{p}, f_{p}\right) \rightarrow X_{p}
$$

be a sequence of basic double links. Then there exists another sequence of basic double links

$$
X=X_{0}:\left(g_{1}, 1\right) \rightarrow Y_{1}:\left(g_{2}, 1\right) \rightarrow \cdots \rightarrow Y_{h-1}:\left(g_{h}, 1\right) \rightarrow Y_{h}
$$

such that

(1) $g_{1} \leq g_{2} \leq \cdots \leq g_{h}$

(2) $Y_{h}$ and $X_{p}$ have the same postulation and are in the same shift of the same even liaison class.

Proof. The fact that the new sequence can be performed with linear forms is Corollary 3.8. So, without loss of generality we can assume that we have a sequence of basic double links of the form

$$
X=X_{0}:\left(b_{1}, 1\right) \rightarrow X_{1}:\left(b_{2}, 1\right) \rightarrow \cdots \rightarrow X_{h-1}:\left(b_{h}, 1\right) \rightarrow X_{h} .
$$

We now prove the lemma by induction on $h$. For $h=1$, there is nothing to prove. Let us suppose $h>1$. We can assume by induction that $b_{i} \geq b_{i-1}$ for $i \leq h-1$. If $b_{h} \geq b_{h-1}$, then the proof is complete.

If $b_{h}<b_{h-1}$, the bulk of the proof rests in showing that the postulation (and of course the shift) is not changed if we replace $\left(\cdots b_{h-1}, b_{h}\right)$ by $\left(\cdots b_{h}-\right.$ $\left.1, b_{h-1}+1\right)$. 
So assume that $b_{h}<b_{h-1}$. We first claim that

$$
h^{0}\left(\mathbf{P}^{n}, \mathcal{I}_{X_{h-2}}\left(b_{h}-1\right)\right) \neq 0
$$

(so in fact such a "flip" is even possible). In fact, let

$$
0 \rightarrow F \rightarrow G \rightarrow \mathscr{I}_{X_{h-2}} \rightarrow 0
$$

be a locally free resolution of $\mathcal{I}_{X_{h-2}}$, with $h^{1}\left(\mathbf{P}^{n}, F(t)\right)=0$ for every $t$ and $G$ direct sum of the line bundles. A resolution of $\mathscr{I}_{X_{h-1}}$ is then

$$
0 \rightarrow F(-1) \oplus \mathscr{O}_{\mathbf{P}^{n}}\left(-b_{h-1}-1\right) \oplus A \rightarrow G(-1) \oplus \mathscr{O}_{\mathbf{p}^{n}}\left(-b_{h-1}\right) \oplus A \rightarrow \mathscr{I}_{X_{h-1}} \rightarrow 0
$$

where $A$ is a line bundle. (Apply the mapping cone procedure of [PS] twice.)

Since $X_{h-1}$ is contained by hypothesis in a hypersurface of degree $b_{h}$, we have

$$
\begin{aligned}
0< & h^{0}\left(\mathbf{P}^{n}, \mathscr{I}_{X_{h-1}}\left(b_{h}\right)\right) \\
= & h^{0}\left(\mathbf{P}^{n}, G\left(b_{h}-1\right)\right)+h^{0}\left(\mathbf{P}^{n}, \mathscr{O}_{\mathbf{P}^{n}}\left(b_{h}-b_{h-1}\right)\right) \\
& -h^{0}\left(\mathbf{P}^{n}, F\left(b_{h}-1\right)\right)-h^{0}\left(\mathbf{P}^{n}, \mathscr{O}_{\mathbf{P}^{n}}\left(b_{h}-b_{h-1}-1\right)\right) \\
= & h^{0}\left(\mathbf{P}^{n}, G\left(b_{h}-1\right)\right)-h^{0}\left(\mathbf{P}^{n}, F\left(b_{h}-1\right)\right)
\end{aligned}
$$

since $b_{h}<b_{h-1}$. Hence

$$
h^{0}\left(\mathbf{P}^{n}, \mathscr{I}_{X_{h-2}}\left(b_{h}-1\right)\right)=h^{0}\left(\mathbf{P}^{n}, G\left(b_{h}-1\right)\right)-h^{0}\left(\mathbf{P}^{n}, F\left(b_{h}-1\right)\right)>0,
$$

proving the claim.

This fact guarantees that we can perform a basic double link

$$
X_{h-2}:\left(b_{h}-1,1\right) \rightarrow Y_{h-1} .
$$

By construction $Y_{h-1}$ is contained in a hypersurface of degree $b_{h}-1$, and since $b_{h-1}+1>b_{h}-1$, it is also contained in a hypersurface of degree $b_{h-1}+1$. Hence we can perform a basic double link

$$
Y_{h-1}:\left(b_{h-1}+1,1\right) \rightarrow Y_{h} .
$$

Clearly $Y_{h}$ and $X_{h}$ are in the same shift of the same even liaison class. We now claim that $Y_{h}$ and $X_{h}$ have the same postulation.

In fact, from $(*)$ we get resolutions for $X_{h}$ and $Y_{h}$ (using [PS]):

$$
\begin{aligned}
0 \rightarrow F(-2) \oplus \mathscr{O}_{\mathbf{P}^{n}}\left(-b_{h-1}-2\right) \oplus \mathscr{O}_{\mathbf{P}^{n}}\left(-b_{h}-1\right) \oplus B \\
\rightarrow G(-2) \oplus \mathscr{O}_{\mathbf{P}^{n}}\left(-b_{h-1}-1\right) \oplus \mathscr{O}_{\mathbf{P}^{n}}\left(-b_{h}\right) \oplus B \rightarrow \mathscr{I}_{X_{h}} \rightarrow 0
\end{aligned}
$$

and

$$
\begin{aligned}
0 \rightarrow F(-2) & \oplus \mathscr{O}_{\mathbf{P}^{n}}\left(-\left(b_{h}-1\right)-2\right) \oplus \mathscr{O}_{\mathbf{P}^{n}}\left(-\left(b_{h-1}+1\right)-1\right) \oplus C \\
& \rightarrow G(-2) \oplus \mathscr{O}_{\mathbf{P}^{n}}\left(-\left(b_{h}-1\right)-1\right) \oplus \mathscr{O}_{\mathbf{P}^{n}}\left(-\left(b_{h-1}+1\right)\right) \oplus C \rightarrow \mathscr{I}_{Y_{h}} \rightarrow 0
\end{aligned}
$$

with $B$ and $C$ direct sums of line bundles. Hence $Y_{h}$ and $X_{h}$ have the same postulation. 
Now, if $b_{h}-1 \geq b_{h-2}$, then the proof is complete. Otherwise, we can repeat this argument until we reach the first $k$ such that $b_{h}-k \geq b_{h-k-1}$, thus getting a sequence of basic double links.

$$
\begin{aligned}
X=X_{0}:\left(b_{1}, 1\right) & \rightarrow X_{1}:\left(b_{2}, 1\right) \rightarrow \cdots \rightarrow X_{h-k-2}:\left(b_{h-k-1}, 1\right) \\
& \rightarrow X_{h-k-1}:\left(b_{h}-k, 1\right) \rightarrow Y_{h-k}:\left(b_{h-k}+1,1\right) \\
& \rightarrow Y_{h-k+1}:\left(b_{h-k+1}+1,1\right) \rightarrow \cdots \rightarrow Y_{h-1}:\left(b_{h-1}+1,1\right) \rightarrow Y_{h}
\end{aligned}
$$

where $Y_{h}$ has the same postulation as that of $X_{h}$. Simply observe that after every "flip" the postulation of the resulting scheme is the same as that of the original one at that level, so the remaining basic double links can still be done. But now we have

$$
b_{1} \leq b_{2} \leq \cdots \leq b_{h-k-1} \leq b_{h}-k \leq b_{h-k}+1 \leq b_{h-k+1}+1 \leq \cdots \leq b_{h-1}+1 .
$$

Corollary 5.3. Let $\mathscr{L}$ be an even liaison class in codimension 2 satisfying the LR-property. Let $X \in \mathscr{L}^{0}$ and let $X^{\prime} \in \mathscr{L}^{h}$. Let

$$
X=X_{0}:\left(b_{1}, f_{1}\right) \rightarrow X_{1}:\left(b_{2}, f_{2}\right) \rightarrow \cdots \rightarrow X_{p-1}:\left(b_{p}, f_{p}\right) \rightarrow X_{p}
$$

be a sequence of basic double links where $X_{p} \in \mathscr{L}^{h}$ has the same postulation as $X^{\prime}$. Let $s=\alpha(X)$ (see Definition 4.2). Then there exists another sequence of basic double links

$$
X=Y_{0}:(s, b) \rightarrow Y_{1}:\left(g_{2}, 1\right) \rightarrow \cdots \rightarrow Y_{r-1}:\left(g_{r}, 1\right) \rightarrow Y_{r}
$$

where

(1) $b \geq 0, s<g_{2}<g_{3}<\cdots<g_{r}$, and $b+r-1=h$;

(2) $X_{p}$ and $Y_{r}$ have the same postulation (as that of $X^{\prime}$ ) and are in the same shift of $\mathscr{L}$.

Moreover, the sequence $\left(b ; g_{2}, \ldots, g_{r}\right)$ is uniquely determined by $X^{\prime}$.

Proof. We have seen that it is enough to consider a sequence of basic double links of the form $\left(b_{i}, 1\right)$, and that a sequence of the form

$$
X=X_{0}:(s, 1) \rightarrow X_{1}:(s, 1) \rightarrow \cdots \rightarrow X_{b-1}:(s, 1) \rightarrow X_{h}
$$

is equivalent to a single basic double link $X=X_{0}:(s, b) \rightarrow Y_{1}$. We have also seen that for any pair of basic double links $X_{i-2}:\left(b_{i-1}, 1\right) \rightarrow X_{i-1}:\left(b_{i}, 1\right) \rightarrow$ $X_{i}$, then whenever it is actually possible to do a pair of basic double links $X_{i-2}:\left(b_{i}-1,1\right) \rightarrow Y_{i-1}:\left(b_{i-1}+1,1\right) \rightarrow Y_{i}$, we are guaranteed that $Y_{i}$ and $X_{i}$ have the same postulation and are in the same shift of the same even liaison class.

We first prove the existence of the special sequence of basic double links described in the corollary. We proceed by induction, the case of just one basic double link being trivial. By Lemma 5.2, we may begin with a sequence of basic double links

$$
X=X_{0}:\left(b_{1}, 1\right) \rightarrow X_{1}:\left(b_{2}, 1\right) \rightarrow \cdots \rightarrow X_{h-1}:\left(b_{h}, 1\right) \rightarrow X_{h}
$$


where $b_{1} \leq \cdots \leq b_{h}$. In fact, by induction one of the following holds:

(a) $s=b_{1}=\cdots=b_{h}$ (then done),

(b) $b_{h-1}<b_{h}$ (then done), or

(c) $b_{h-2}<b_{h-1}=b_{h}$.

For (c), $b_{h-2} \leq b_{h-1}-1=b_{h}-1$. By the construction of basic double linkage, $h^{0}\left(\mathcal{J}_{X_{h-2}}\left(b_{h-2}\right)\right) \neq 0$ so also $h^{0}\left(\mathscr{J}_{X_{h-2}}\left(b_{h}-1\right)\right) \neq 0$. Then our sequence of basic double links is equivalent to

$$
\cdots X_{h-2}:\left(b_{h}-1,1\right) \rightarrow Y_{h-1}:\left(b_{h-1}+1,1\right) \rightarrow Y_{h} .
$$

We now have $b_{h-2} \leq b_{h}-1<b_{h-1}+1$. The possibilities are

$\left(\mathbf{a}^{\prime}\right) b_{h-2}=b_{h}-1=s$ (then done),

( $\left.\mathbf{b}^{\prime}\right) b_{h-2}<b_{h}-1$ (then done), or

(c') $b_{h-3}<b_{h-2}=b_{h}-1$.

We continue by descending induction, and this proves the existence of the desired sequence of basic double links. The condition $b+r-1=h$ of (1) and condition (2) are immediate. It remains to prove that this sequence is uniquely determined by the cohomology of $X^{\prime}$.

Suppose that there are two sequences of basic double links

$$
\begin{aligned}
& X=Y_{0}:(s, b) \rightarrow Y_{1}:\left(g_{2}, 1\right) \rightarrow \cdots \rightarrow Y_{r-1}:\left(g_{r}, 1\right) \rightarrow Y_{r} \text { and } \\
& X=Y_{0}:(s, c) \rightarrow Z_{1}:\left(h_{2}, 1\right) \rightarrow \cdots \rightarrow Z_{t-1}:\left(h_{t}, 1\right) \rightarrow Z_{t},
\end{aligned}
$$

with $g_{2}<g_{3}<\cdots<g_{r}$ and $h_{2}<h_{3}<\cdots<h_{t}$, and that $Y_{r}$ and $Z_{t}$ have the same postulation and lie in the same shift. First of all, the integer $b$ must be equal to $c$ and $r$ equal to $t$. In fact, $s+r-1=\alpha\left(Y_{r}\right)=\alpha\left(Z_{t}\right)=s+t-1$; since moreover $b+r-1=h=c+t-1$ we get $b=c$ and $r=t$. Hence we can suppose that $Y_{1}$ and $Z_{1}$ have two locally free resolutions

$$
0 \rightarrow F \rightarrow G \rightarrow \mathscr{I}_{Y_{1}} \rightarrow 0, \quad 0 \rightarrow F_{1} \rightarrow G_{1} \rightarrow \mathscr{I}_{Z_{1}} \rightarrow 0
$$

such that for every $t$ we have

$$
h^{0}\left(\mathbf{P}^{n}, F(t)\right)-h^{0}\left(\mathbf{P}^{n}, G(t)\right)=h^{0}\left(\mathbf{P}^{n}, F_{1}(t)\right)-h^{0}\left(\mathbf{P}^{n}, G_{1}(t)\right)
$$

since $Y_{1}$ and $Z_{1}$ have the same postulation.

But now $Y_{r}$ and $Z_{r}$ have resolutions of the form

$$
\begin{aligned}
0 & \rightarrow \bigoplus_{i=2}^{r} \mathscr{O}_{\mathbf{p}^{n}}\left(-g_{i}-r+i-1\right) \oplus F(-r+1) \oplus T \\
& \rightarrow \bigoplus_{i=2}^{r} \mathscr{O}_{\mathbf{p}^{n}}\left(-g_{i}-r+i\right) \oplus G(-r+1) \oplus T \rightarrow \mathscr{I}_{Y_{r}} \rightarrow 0, \\
0 & \rightarrow \bigoplus_{i=2}^{r} \mathscr{O}_{\mathbf{p}^{n}}\left(-h_{i}-r+i-1\right) \oplus F_{1}(-r+1) \oplus S \\
& \rightarrow \bigoplus_{i=2}^{r} \mathscr{Q}_{\mathbf{p}^{n}}\left(-h_{i}-r+i\right) \oplus G_{1}(-r+1) \oplus S \rightarrow \mathscr{I}_{Z_{r}} \rightarrow 0,
\end{aligned}
$$


where $T$ and $S$ are direct sums of line bundles (this will be a standard procedure when we use sequences of basic double links; see also Lemma 5.2 and Proposition 5.10 below). Note that $-g_{i}-r+i \geq-g_{i+1}-r+i+1$ (since $\left.g_{i} \leq g_{i+1}-1\right)$, and the same thing is true for the $h_{i}$ 's. Suppose that $g_{2} \leq h_{2}$, and that $g_{3}=g_{2}+1, g_{4}=g_{3}+1, \ldots, g_{v}=g_{v-1}+1, g_{v+1}>g_{v}+1$, with $v \geq 2$. Then we have

$$
h^{0}\left(\mathbf{P}^{n}, \mathscr{J}_{Y_{r}}\left(g_{2}+r-2\right)\right)=h^{0}\left(\mathbf{P}^{n}, G\left(g_{2}-1\right)\right)-h^{0}\left(\mathbf{P}^{n}, F\left(g_{2}-1\right)\right)+(v+1)
$$

and

$$
\begin{aligned}
h^{0}\left(\mathbf{P}^{n}, \mathcal{I}_{Z_{r}}\left(g_{2}\right.\right. & +r-2))=h^{0}\left(\mathbf{P}^{n}, G_{1}\left(g_{2}-1\right)\right)-h^{0}\left(\mathbf{P}^{n}, F_{1}\left(g_{2}-1\right)\right) \\
& +\sum_{i} h^{0}\left(\mathbf{P}^{n}, \mathscr{O}_{\mathbf{P}^{n}}\left(-h_{i}+i+g_{2}-2\right)\right) .
\end{aligned}
$$

Since $h^{0}\left(\mathbf{P}^{n}, \mathscr{F}_{Y_{r}}\left(g_{2}+r-2\right)\right)=h^{0}\left(\mathbf{P}^{n}, \mathscr{I}_{Z_{r}}\left(g_{2}+r-2\right)\right)$ and $h^{0}\left(\mathbf{P}^{n}, G_{1}\left(g_{2}-1\right)\right)-$ $h^{0}\left(\mathbf{P}^{n}, F_{1}\left(g_{2}-1\right)\right)=h^{0}\left(\mathbf{P}^{n}, G\left(g_{2}-1\right)\right)-h^{0}\left(\mathbf{P}^{n}, F\left(g_{2}-1\right)\right)$, it follows that $h_{2}=$ $g_{2}, h_{3}=g_{3}, \ldots, h_{v}=g_{v}$. Then repeat this argument with $\min \left\{g_{v+1}, h_{v+1}\right\}$ in place of $g_{2}$ above, and so on.

Remark 5.4. In this way we have a "standard form" for describing the cohomology of a subscheme belonging to an even liaison class with the LR-property. This is useful when we want to have a control on the degrees of the hypersurfaces occurring in a sequence of basic double links. When we have such a standard form, we make the convention that if there are no $g_{i}$ 's, we assume $r=1$.

Example 5.5. It is natural to ask whether the condition that our schemes lie in the same shift of the same even liaison class is redundant. That is, does specifying the postulation (equivalently the Hilbert function) and the even liaison class automatically determine the shift? Notice that if we allow the shifts to differ, we no longer have Proposition 3.1 and Corollary 3.4, so it makes a difference whether we consider the postulation of the hyperplane section or that of the scheme itself.

In either case, it is not hard to see that the condition of having the same shift is not redundant. For both cases, our example will be in the (even) liaison class $\mathscr{L}$ of two skew lines in $\mathbf{P}^{3}$. Let $C$ be a pair of skew lines. Then $\alpha(C)=2$. We first consider the former case. Using Proposition 1.11 of [BM2] one can check that the sequences of basic double links

$$
\begin{aligned}
& C=C_{0}:(2,4) \rightarrow C_{1}:(4,1) \rightarrow C_{2}:(6,1) \rightarrow C_{3}, \\
& C=D_{0}:(2,3) \rightarrow D_{1}:(4,1) \rightarrow D_{2}:(8,1) \rightarrow D_{3}
\end{aligned}
$$

give curves whose hyperplane sections have the same postulation, but the first is in the shift $h=6$ while the second has $h=5$. Of course, this necessarily implies that the curves themselves have different postulations in this case. 
On the other hand, consider the sequences of basic double links

$$
\begin{aligned}
& C=C_{0}:(2,2) \rightarrow C_{1}:(4,1) \rightarrow C_{2}:(5,1) \rightarrow C_{3}:(6,1) \rightarrow C_{4}:(9,1) \rightarrow C_{5}, \\
& C=D_{0}:(2,1) \rightarrow D_{1}:(5,1) \rightarrow D_{2}:(6,1) \rightarrow D_{3}:(7,1) \rightarrow D_{4}:(8,1) \rightarrow D_{5} .
\end{aligned}
$$

One can check using [BM2, Proposition 1.11] (and some further elementary calculations) or Corollary 5.9 below that $C_{5}$ and $D_{5}$ are curves with the same postulation, but clearly $C_{5}$ lies in shift $h=6$ while $D_{5}$ lies in shift $h=5$.

Notation 5.6. Let $X \subset \mathbf{P}^{n}$ be a two-codimensional subscheme. We define the function $F_{X}:\{(0,1, \ldots, n-1) \times \mathbf{Z} \rightarrow \mathbf{Z}\}$ by $F_{X}(i, t)=h^{i}\left(\mathbf{P}^{n}, \mathscr{J}_{X}(t)\right)$.

If $X$ and $Y$ are two-codimensional subschemes of $\mathbf{P}^{n}$, we will say briefly that they have the same cohomology (dimensionality) if $F_{X}=F_{Y}$.

Corollary 5.7. Let $\mathscr{L}$ be an even liaison class of two-codimensional subschemes of $\mathbf{P}^{n}$ with the LR-property, and let $M$ be minimal in $\mathscr{L}$. Then the elements of $\mathscr{L}$ are distributed in disjoint nonvoid subsets $\mathscr{F}_{\left(b ; g_{2}, g_{3}, \ldots, g_{r}\right)}$, where $b \geq 0$, $g_{r}>g_{r-1}>\cdots>g_{2}>\alpha(M)$, such that any two elements of the same subset have the same cohomology (dimensionally).

Proof. Let us call $\mathscr{F}_{\left(b ; g_{2}, g_{3}, \ldots, g_{r}\right)}$ the set of subschemes $X \in \mathscr{L}$ having the same cohomology of a subscheme obtained from $M$ via a sequence of basic double links

$$
M=Y_{0}:(\alpha(M), b) \rightarrow Y_{1}:\left(g_{2}, 1\right) \rightarrow \cdots \rightarrow Y_{r-1}:\left(g_{r}, 1\right) \rightarrow Y_{r} .
$$

Then the corollary follows from Corollary 5.3 and the proof of Proposition 3.11 .

Remark 5.8. Of course, there might exist a deformation between subschemes lying in different shifts of the same even liaison class, through subschemes of different liaison classes. In fact, Example 5.6 suggests that this is actually possible.

Corollary 5.9. Let $\mathscr{L}$ be an even liaison class of two-codimensional subschemes of $\mathbf{P}^{n}$ with the LR-property, let $M$ be minimal in $\mathscr{L}$, and let $X \in \mathscr{F}_{\left(b ; g_{2}, g_{3}, \ldots, g_{r}\right)}$. Then

$$
\begin{aligned}
F_{X}(0, t)= & F_{M}(0, t-b-r+1)+h^{0}\left(\mathbf{P}^{n}, \mathscr{O}_{\mathbf{p}^{n}}(t-\alpha-r+1)\right) \\
& -h^{0}\left(\mathbf{P}^{n}, \mathscr{O}_{\mathbf{p}^{n}}(t-\alpha-b-r+1)\right) \\
& +\sum_{i=2}^{r} h^{0}\left(\mathbf{P}^{n}, \mathscr{O}_{\mathbf{P}^{n}}\left(t-g_{i}-r+i\right)\right) \\
& -\sum_{i=2}^{r} h^{0}\left(\mathbf{P}^{n}, \mathscr{O}_{\mathbf{P}^{n}}\left(t-g_{i}-r+i-1\right)\right)
\end{aligned}
$$

and $F_{X}(i, t)=F_{M}(i, t-p-r+1)$ if $0<i<n-1$.

Proof. Suppose that $M$ has a resolution

$$
0 \rightarrow F \rightarrow G \rightarrow \mathscr{I}_{M} \rightarrow 0
$$


with $G$ direct sum of line bundles and $h^{1}\left(\mathbf{P}^{n}, F(t)\right)=0$ for every $t$. Let $\alpha=\alpha(M)$, that is, the minimal degree of a hypersurface containing $M$.

The first result follows from an easy computation, knowing that $X$ has the same cohomology as $Y_{r}$, obtained from $M$ via a sequence of basic double links

$$
M=Y_{0}:(\alpha, b) \rightarrow Y_{1}:\left(g_{2}, 1\right) \rightarrow \cdots \rightarrow Y_{r-1}:\left(g_{r}, 1\right) \rightarrow Y_{r}
$$

and that therefore $Y_{r}$ has a resolution of the form

$$
\begin{aligned}
0 & \rightarrow \bigoplus_{i=2}^{r} \mathscr{O}_{\mathbf{p}^{n}}\left(-g_{i}-r+i-1\right) \oplus F(-b-r+1) \oplus T \oplus \mathscr{O}_{\mathbf{P}^{n}}(-\alpha-b-r+1) \\
& \rightarrow \bigoplus_{i=2}^{r} \mathscr{O}_{\mathbf{P}^{n}}\left(-g_{i}-r+i\right) \oplus G(-b-r+1) \oplus T \oplus \mathscr{O}_{\mathbf{P}^{n}}(-\alpha-r+1) \rightarrow \mathscr{I}_{Y_{r}} \rightarrow 0
\end{aligned}
$$

where $T$ is a direct sum of line bundles. The second assertion follows from the fact that $X$ is in $\mathscr{L}^{p+r-1}$.

Proposition 5.10. Let $1 \leq b_{1} \leq b_{2} \leq \cdots \leq b_{p}$ be a nondecreasing sequence of integers. Then there exists an arithmetically Cohen-Macaulay two-codimensional subscheme of $\mathbf{P}^{n}, C=C\left(b_{1}, b_{2}, \ldots, b_{p}\right)$, having a locally free resolution

$0 \rightarrow \bigoplus_{i=1}^{P} \mathscr{O}_{\mathbf{P}^{n}}\left(-b_{i}-p+i-1\right) \oplus B \rightarrow \bigoplus_{i=1}^{p} \mathscr{O}_{\mathbf{P}^{n}}\left(-b_{i}-p+i\right) \oplus B \oplus \mathscr{O}_{\mathbf{P}^{n}}(-p) \rightarrow \mathscr{I}_{C} \rightarrow 0$

where $B$ is a direct sum of line bundles. Moreover, $C$ is contained in a hypersurface of degree $=\min \left\{b_{p}, p\right\}$.

Proof. By induction on $p$. If $p=1$, then take as $C=C\left(b_{1}\right)$ the complete intersection of a hyperplane and a hypersurface of degree $b_{1}$. Then

$$
0 \rightarrow \mathscr{O}_{\mathbf{P}^{n}}\left(-b_{1}-1\right) \rightarrow \mathscr{O}_{\mathbf{P}^{n}}\left(-b_{1}\right) \oplus \mathscr{O}_{\mathbf{P}^{n}}(-1) \rightarrow \mathscr{I}_{C} \rightarrow 0
$$

is the well-known locally free resolution of $\mathscr{I}_{C}$.

Now, let $D=C\left(b_{1}, b_{2}, \ldots, b_{p-1}\right)$, and let

$0 \rightarrow \bigoplus_{i=1}^{p-1} \mathscr{O}_{\mathbf{P}^{n}}\left(-b_{i}-p+i\right) \oplus A \rightarrow \bigoplus_{i=1}^{p-1} \mathscr{O}_{\mathbf{p}^{n}}\left(-b_{i}-p+i+1\right) \oplus A \oplus \mathscr{O}_{\mathbf{P}^{n}}(-p+1) \rightarrow \mathscr{I}_{D} \rightarrow 0$

be its resolution. Since by induction $D$ is contained in a hypersurface of degree $b_{p-1}$, there also exists a hypersurface $\Sigma$ of degree $b_{p} \geq b_{p-1}$ containing $D$. Perform a basic double link of $D$ via $\Sigma$ and a general hyperplane $H$, and call $C$ the (reducible) subscheme thus obtained. We claim that $C=C\left(b_{1}, b_{2}, \ldots, b_{p}\right)$. In fact, $C$ is obtained from $D$ with two linkages: first, link $D$ to $Y$ using $\Sigma$ and a hypersurface $V$ of degree $v>>0$, and then link $Y$ to $C$ by using 
$V H$ and $\Sigma$. Trivially, $Y$ and $C$ are arithmetically Cohen-Macaulay twocodimensional subschemes having locally free resolutions

$$
\begin{aligned}
0 & \rightarrow \bigoplus_{i=1}^{p-1} \mathscr{O}_{\mathbf{P}^{n}}\left(b_{i}+p-i-1-b_{p}-v\right) \oplus A^{\vee}\left(-b_{p}-v\right) \oplus \mathscr{O}_{\mathbf{P}^{n}}\left(p-1-b_{p}-v\right) \\
\rightarrow & \bigoplus_{i=1}^{p-1} \mathscr{O}_{\mathbf{P}^{n}}\left(b_{i}+p-i-b_{p}-v\right) \oplus A^{\vee}\left(-b_{p}-v\right) \\
& \oplus \mathscr{O}_{\mathbf{P}^{n}}\left(-b_{p}\right) \oplus \mathscr{O}_{\mathbf{P}^{n}}(-v) \rightarrow \mathscr{I}_{Y} \rightarrow 0
\end{aligned}
$$

and

$$
\begin{aligned}
0 \rightarrow & \bigoplus_{i-1}^{p-1} \mathscr{O}_{\mathbf{p}^{n}}\left(-b_{i}-p+i-1\right) \oplus \mathscr{O}_{\mathbf{P}^{n}}\left(-b_{p}-1\right) \oplus\left(A(-1) \oplus \mathscr{O}_{\mathbf{P}^{n}}(-v-1)\right) \\
\rightarrow & \bigoplus_{i=1}^{p-1} \mathscr{O}_{\mathbf{P}^{n}}\left(-b_{i}-p+i\right) \oplus \mathscr{O}_{\mathbf{P}^{n}}\left(-b_{p}\right) \oplus\left(A(-1) \oplus \mathscr{O}_{\mathbf{P}^{n}}(-v-1)\right) \\
& \oplus \mathscr{O}_{\mathbf{P}^{n}}(-p) \rightarrow \mathscr{I}_{C} \rightarrow 0
\end{aligned}
$$

and $B=\left(A(-1) \oplus \mathscr{O}_{\mathbf{P}^{n}}(-v-1)\right)$ is a direct sum of line bundles. By construction, $C$ is contained in a hypersurface of degree $b_{p}$ and also in a hypersurface of degree $p$, thus concluding the proof.

For the following theorem we adopt the convention that the void set is an arithmetically Cohen-Macaulay subscheme of $\mathbf{P}^{n}$ (for example, residual to a complete intersection).

Theorem 5.11. Let $X$ be a two-codimensional subscheme of $\mathbf{P}^{n}$ and let $X_{h}$ be the result of applying any sequence of basic double links to $X$. Then $X_{h}$ specializes to the liaison addition of $X$ with a suitable arithmetically CohenMacaualy subscheme (possibly void).

Proof. Thanks to Lemma 5.2, without loss of generality we can suppose $X=$ $X_{0}:\left(b_{1}, 1\right) \rightarrow X_{1}:\left(b_{2}, 1\right) \rightarrow \cdots \rightarrow X_{h-1}:\left(b_{h}, 1\right) \rightarrow X_{h}$ where $b_{i} \geq b_{i-1}$ for every $i$.

If $b_{1}=b_{2}=\cdots=b_{h}$, then $X_{h}$ itself is the liaison addition of $X$ and the void set via hypersurfaces of degrees $b_{1}$ and $h$. Otherwise, let $m=$ $\min \left\{i \mid b_{i}>b_{1}\right\}$, and let

$$
C=C\left(b_{m}-b_{1}, b_{m+1}-b_{1}, \ldots, b_{h}-b_{1}\right) .
$$

Thanks to Proposition 5.9, $C$ is contained in a hypersurface of degree $h-m+$ $1 \leq h$.

Let

$$
0 \rightarrow F \rightarrow G \rightarrow \mathscr{I}_{X} \rightarrow 0
$$


be a resolution of $X$, with $G$ a direct sum of line bundles and $F$ locally free such that $H_{*}^{1}\left(\mathbf{P}_{n}, F\right)=0$. A locally free resolution of $\mathscr{I}_{X_{h}}$ is then of the form

$$
\begin{aligned}
0 & \rightarrow \bigoplus_{i=1}^{h} \mathscr{O}_{\mathbf{P}^{n}}\left(-b_{i}-h-1+i\right) \oplus F(-h) \oplus S \\
& \rightarrow \bigoplus_{i=1}^{h} \mathscr{O}_{\mathbf{P}^{n}}\left(-b_{i}-h+i\right) \oplus G(-h) \oplus S \rightarrow \mathscr{I}_{X_{h}} \rightarrow 0
\end{aligned}
$$

where $S$ is a direct sum of line bundles.

For $1<i<m,-b_{i}-h+i-1=-b_{i+1}-h+(i-1)$, since $b_{i}=b_{i-1}$. Hence the previous resolution can be written

$$
\begin{aligned}
0 & \rightarrow \bigoplus_{i=m}^{h} \mathscr{O}_{\mathbf{P}^{n}}\left(-b_{i}-h-1+i\right) \oplus F(-h) \oplus T \oplus \mathscr{O}_{\mathbf{P}^{n}}\left(-b_{1}-h\right) \\
& \rightarrow \bigoplus_{i=m}^{h} \mathscr{O}_{\mathbf{P}^{n}}\left(-b_{i}-h+i\right) \oplus \mathscr{O}_{\mathbf{P}^{n}}\left(-b_{1}-h+m-1\right) \oplus G(-h) \oplus T \rightarrow \mathscr{I}_{X_{h}} \rightarrow 0
\end{aligned}
$$

where $T$ is a direct sum of line bundles (recall that $b_{m-1}=b_{1}$ ).

Now let us perform the liaison addition of $X$ and $C$ via hypersurfaces of degrees $b_{1}$ and $h$ respectively, and call $Z$ the resulting subscheme. Recall that $C$ has a resolution of the form

$$
\begin{aligned}
0 & \rightarrow \bigoplus_{i=m}^{h} \mathscr{O}_{\mathbf{p}^{n}}\left(-b_{i}-h+b_{1}+i-1\right) \oplus A \\
& \rightarrow \bigoplus_{i=m}^{h} \mathscr{O}_{\mathbf{P}^{n}}\left(-b_{i}-h+b_{1}+i\right) \oplus \mathscr{O}_{\mathbf{P}^{n}}(-h+m-1) \oplus A \rightarrow \mathscr{I}_{C} \rightarrow 0 .
\end{aligned}
$$

Hence by Proposition 2.7, $Z$ has a resolution of the form

$$
\begin{aligned}
0 & \rightarrow \bigoplus_{i=m}^{h} \mathscr{O}_{\mathbf{p}^{n}}\left(-b_{i}-h+i-1\right) \oplus \mathscr{O}_{\mathbf{P}^{n}}\left(-b_{1}-h\right) \oplus A\left(-b_{1}\right) \oplus F(-h) \\
\rightarrow & \bigoplus_{i=m}^{h} \mathscr{C}_{\mathbf{P}^{n}}\left(-b_{i}-h+i\right) \oplus \mathscr{O}_{\mathbf{P}^{n}}\left(-b_{1}-h+m-1\right) \\
& \oplus A\left(-b_{1}\right) \oplus G(-h) \rightarrow \mathscr{I}_{Z} \rightarrow 0 .
\end{aligned}
$$

It follows that $Z$ and $X_{h}$ have the same postulation, and since $Z$ and $X_{h}$ are in the same shift of the same even liaison class we have that there exists a deformation from $Z$ to $X_{h}$.

This gives us a different point of view for studying the structure of an even liaison class:

Corollary 5.12. Let $\mathscr{L}$ be an even liaison class in codimension two with the $L R$ property, and let $X \in \mathscr{L}^{0}$. Then every $Z \in \mathscr{L}$ specializes to the liaison addition of $X$ and some arithmetically Cohen-Macaulay subscheme of $\mathbf{P}^{n}$. 
Example 5.13. We collect our results in the description of an example. We concentrate on surfaces in $\mathbf{P}^{4}$, but exactly the same is true for threefolds in $\mathbf{P}^{5}$ and for $(n-2)$-dimensional subschemes of $\mathbf{P}^{n}$ in general. Let $X$ be a surface in $\mathbf{P}^{4}$. If $X$ is arithmetically Cohen-Macaulay, then $h^{1}\left(\mathbf{P}^{4}, \mathscr{I}_{X}(t)\right)=$ $0=h^{2}\left(\mathbf{P}^{4}, \mathscr{I}_{X}(t)\right)$ for every $t$. These surfaces are well known; that is to say, we know precisely all the possible postulations, degrees, arithmetic genera, sectional genera and so on [GP, E, B-MR].

The simplest nonarithmetically Cohen-Macaulay case is a surface $X$ with only one group $H^{1}\left(\mathbf{P}^{4}, \mathscr{J}_{X}(t)\right)$ or $H^{2}\left(\mathbf{P}^{4}, \mathscr{I}_{X}(t)\right)$ nonzero. As we saw in $\S 4$, the liaison class of $X$ satisfies the LR-property. Let $M_{(1, p)}$ be a minimal surface in the liaison class of surfaces with only one group $H^{1}\left(\mathbf{P}^{4}, \mathscr{I}_{X}(t)\right)$ nonzero and p-dimensional, and all $H^{2}\left(\mathbf{P}^{4}, \mathscr{I}_{X}(t)\right)=0$; we define $M_{(2, p)}$ in a similar way. Note that we can explicitly compute the cohomology function $F_{M_{(i, p)}}$ since we have an explicit locally free resolution for the sheaf $\mathscr{I}_{M_{(i, p)}}$. For instance, $M_{(1,1)}$ is the general projection to $\mathbf{P}^{4}$ of the Veronese surface in $\mathbf{P}^{5}$ (or a surface with the same cohomology), and $\operatorname{deg}\left(M_{(1,1)}\right)=4, \pi\left(M_{(1,1)}\right)=0, p_{a}\left(M_{(1,1)}\right)=$ 0 . The rest can be computed using Liaison Addition (see Example 4.11) and the fact that basic double linkage is also preserved under hyperplane sections (Lemma 3.5).

Collecting all results, we can say that the set of all two-codimensional subschemes of $\mathbf{P}^{4}$ with only one intermediate cohomology group different from zero is subdivided into disjoint nonvoid subsets $\mathscr{F}_{\left(i, p, b ; g_{2}, g_{3}, \ldots, g_{r}\right)}$ with $1 \leq i \leq$ $2, p \geq 1, b \geq 0,3 p=\alpha\left(M_{(i, p)}\right)<g_{2}<g_{3}<\cdots<g_{r}$ and two subschemes of the same subset have the same cohomology. Moreover, if $X \in \mathscr{F}_{\left(i, p, b ; g_{2}, g_{3}, \ldots, g_{r}\right)}$, then

$$
\begin{gathered}
\alpha(x)=\alpha\left(M_{(i, p)}\right)+r-1=3 p+r-1, \\
\operatorname{deg}(X)=\operatorname{deg}\left(M_{(i, p)}\right)+\alpha\left(M_{(i, p)}\right) b+\sum g_{i}, \\
p_{a}(X)=h^{2}\left(W, \mathscr{O}_{W^{\prime}}(-r+1)\right)+h^{2}\left(M, \mathscr{O}_{M}(-b-r+1)\right) \\
+\sum_{j=0}^{r-2} h^{2}\left(C_{r-j}, \mathscr{O}_{C_{r-j}}(-j)\right), \\
\pi(X)=\pi(M)+\frac{1}{2}\left(\alpha b^{2}-4 \alpha b+\alpha^{2} b\right)+(b+r-1) \operatorname{deg}(M)+(r-1) b \alpha \\
+\sum_{i=2}^{r}\left[\frac{1}{2}\left(g_{i}^{2}-3 g_{i}\right)+(r-i) g_{i}\right],
\end{gathered}
$$

where $M$ is $M_{(i, p)}, W$ is the complete intersection of hypersurfaces of degrees $\alpha(M)$ and $b$, and $C_{j}$ is the complete intersection of a hyperplane and a hypersurface of degree $g_{j}$, whose cohomologies can be computed in a standard way (remember that we assume that $r=1$ if there are no $g_{i}$ 's), and in general $F_{X}$ can be computed by using Corollary 5.9. 
This provides a complete description of all possible families of two-dimensional subschemes of $\mathbf{P}^{4}$ with only one intermediate cohomology group different from zero.

Notice that a different problem is the determination of families containing a smooth element, or an element of maximal rank (both are open properties): these problems are studied in [C] and [B-MR]; for curves in $\mathbf{P}^{3}$ these liaison classes are studied in [BM1].

\section{QUESTIONS AND COMMENTS}

(1) The most obvious question arising from this work is which even liaison classes possess the LR-property. We conjecture that it holds for every nonarithmetically Cohen-Macaulay codimension two even liaison class in $\mathbf{P}^{n} .{ }^{1}$ More genrally, though, it seems reasonable to hope that it in fact holds for every nonarithmetically Cohen-Macaulay even liaison class of arbitrary codimension.

(2) As a result of Corollary 4.10(a) (or more generally [C]), it follows from [GM1, Corollary 3.9(a)] that a necessary condition for a curve $C \in \mathscr{L}_{3.1}$ to be the hyperplane section of a surface $Y \in \mathscr{L}_{4,1}$ or $\mathscr{L}_{4,2}$ is that it lie on no surface of degree $<3 p-1$. Since curves in $\mathscr{L}_{3,1}$ have Amasaki's lower bound of $2 p$ for the degres of surfaces containing them [A, GM2], this says that any curve in $\mathscr{L}_{3,1}$ lying on a surface of fairly small degree for the liaison class cannot be the hyperplane section of a surface $Y \in \mathscr{L}_{4,1}$ or $\mathscr{L}_{4,2}$.

Two natural questions arise from this. First, if $C \subset \mathbf{P}^{3}$ is arithmetically Buchsbaum, when is $C$ the hyperplane section of an arithmetically Buchsbaum surface $Y$ in $\mathbf{P}^{4}$ ? When is it the intersection in $\mathbf{P}^{n}$ of a 3-plane with a codimension two arithmetically Buchsbaum subscheme? What is the largest $n$ for which this holds? Given C, how many different arithmetically Buchsbaum codimension two even liaison classes in $\mathbf{P}^{n}$ contain such a $Y$ ?

Second, a much more difficult question is to remove the condition of "arithmetically Buchsbaum" above and only ask that $Y$ be locally Cohen-Macaulay. Note that this almost always eliminates the "obvious" first guess of letting $Y$ be a cone over $C$.

(3) We have seen the relation between even liaison classes in codimension two and vector bundles (Theorem 2.2). If $\mathscr{L}$ is an even liaison class and $\mathscr{L}^{*}$ is the residual liaison class, what is the relation between the corresponding vector bundles?

\section{REFERENCES}

[A1] M. Amasaki, On the strcuture of arithmetically Buchsbaum curves in $\mathbf{P}_{k}^{3}$, Publ. Res. Inst. Math. Sci. 20 (1984), 793-837.

[B] G. Boiondi, Irreducible families of curves with fixed cohomology, Arch. Math. (to appear).

1 Note added in proof. This conjecture has recently been proved by the two present authors together with E. Ballico. More recently a different proof for the case of curves in $\mathbf{P}^{3}$ was found by M. Martin-Deschamps and D. Perrin. 
[BM1] G. Bolondi and J. Migliore, Buchsbaum liaison classes, J. Algebra (to appear).

[BM2] _ The Lazarsfeld-Rao and Zeuthen problem for Buchsbaum curves, Rend. Sem. Mat. Univ. Padova (to appear).

[B-MR] G. Bolondi and R. Miro-Roig, Buchsbaum subschemes via hyperplane sections, preprint.

[C] M. Chang, Buchsbaum subvarieties of codimension 2 in $\mathbf{P}^{n}$, preprint.

[DGO] E. Davis, A. V. Geramita and F. Orecchia, Gorenstein algebras and the Cayley-Bacharach theorem, Proc. Amer. Math. Soc. 93 (1985), 593-597.

[E] G. Ellingsrud, Sur le schéma de Hilbert des variétés de codimension 2 dans $\mathbf{p}^{e}$ à cône de Cohen-Macaulay, Ann. Sci. École Norm. Sup. (4) 8 (1975), 423-431.

[GM1] A. V. Geramita and J. Migliore, On the ideal of an arithmetically Buchsbaum curve, J. Pure Appl. Algebra 54 (1988), 215-247.

[GM2] _ _ Generators of the ideal of a Buchsbaum curve, J. Pure Appl. Algebra 58 (1989), 147167.

[GP] L. Gruson and Chr. Peskine, Genre des courbes de l'espace projectif, Algebraic Geometry, Proceedings (Tromso) 1977, Lecture Notes in Math., vol. 687, Springer-Verlag, Berlin, 1978, pp. 31-59.

[H] R. Hartshorne, Algebraic geometry, Springer-Verlag, New York, 1977.

[HU] C. Huneke and B. Ulrich, The structure of linkage, Ann. of Math. 126 (1987), 221-275.

[K] S. Kleiman, Geometry on grassmannians and applications to splitting bundles and smoothing cycles, Inst. Hautes Études Sci. Publ. Math. 36 (1969), 282-298.

[LR] R. Lazarsfeld and P. Rao, Linkage of general curves of large degree, Algebraic GeometryOpen Problems (Ravello 1982), Lecture Notes in Math., vol. 997, Springer-Verlag, Berlin, 1983, pp. 267-289.

[M1] J. Migliore, Geometric invariants for liaison of space curves, J. Algebra 99 (1986), 548-572.

[M2] __, Liaison of a union of skew lines in $\mathbf{P}^{4}$, Pacific J. Math. 130 (1987), 153-170.

[O1] Ch. Okonek, Moduli reflexiver Garben und Flächen von kleinem Grad in $\mathbf{P}^{4}$, Math. Z. 184 (1983), 549-572.

[O2] __ Flächen vom Grad 8 im P4, Math. Z. 191 (1986), 207-223.

[PS] C. Peskine and L. Szpiro, Liaison des variétés algébriques, I, Invent Math. 26 (1974), 271-302.

[R] P. Rao, Liaison equivalence classes, Math. Ann. 258 (1981), 169-173.

[S] P. Schenzel, Notes on liaison and duality, J. Math. Kyoto Univ. 22 (3) (1982), 485-498.

[Sw] Ph. Schwartau, Liaison addition and monomial ideals, Ph.D. Thesis, Brandeis University, 1982.

[SV] J. Stuckrad and W. Vogel, Buchshaum rings and applications, Springer-Verlag, Berlin, 1986.

Dipartimento di Matematica e Fisica, Università di Camerino, I 62032 Camerino (MacERATA), ITALY (Current address of Giorgio Bolondi)

Department of Mathematics, Drew University, Madison, New Jersey 07940

Current address (J. C. Migliore): Department of Mathematics, University of Notre Dame, Notre Dame, Indiana 46556 